\title{
Review of the Data Supporting the Lubrizol Evaluation of Multimedia Impacts Resulting from the Use of PuriNOx Fuel in California
}

\author{
Harry R. Beller \\ Brendan P. Dooher \\ Linda C. Hall \\ David W. Layton \\ Alfredo A. Marchetti \\ Walt W. McNab \\ David W. Rice*
}

\section{September 2003}

\section{Environmental Protection Department Environmental Restoration Program and Division}




\section{DISCLAIMER}

Neither the United States Government nor the University of California nor any of their employees, makes any warranty, express or implied, or assumes any legal liability or responsibility for the accuracy, completeness, or usefulness of any information, apparatus, product, or process disclosed, or represents that its use would not infringe privately owned rights. Reference herein to any specific commercial products, process or service by trade name, trademark, manufacturer, or otherwise, does not necessarily constitute or imply its endorsement, recommendation, or favoring by the United States Government or the University of California. The views and opinions of authors expressed herein do not necessarily state or reflect those of the United States Government or the University of California, and shall not be used for advertising or product endorsement purposes. 


\title{
Review of the Data Supporting the Lubrizol Evaluation of Multimedia Impacts Resulting from the Use of PuriNOx Fuel in California
}

\author{
Harry R. Beller \\ Brendan P. Dooher \\ Linda C. Hall \\ David W. Layton \\ Alfredo A. Marchetti \\ Walt W. McNab \\ David W. Rice*
}

*Expert Panel Chairman and Primary Point of Contact

\section{September 2003}

\section{Environmental Protection Department}

Environmental Restoration Program and Division 


\section{Table of Contents}

I. Executive Summary and Recommendations .......................................................................ES-1

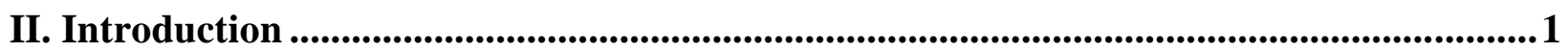

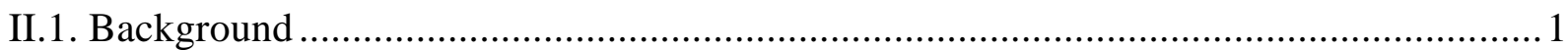

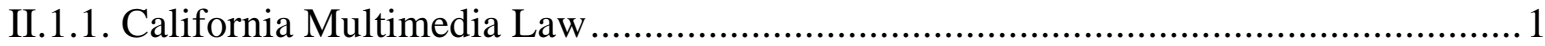

II.1.2. SWRCB Evaluation Criteria .......................................................................

II.1.3. The LLNL Expert Panel's Role.....................................................................

II.2. Summary of the Regulatory Approval Status for PuriNOx ………................................

III. Review of Submitted Data and Identification of Critical Knowledge Gaps......................6

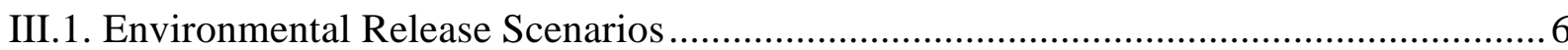

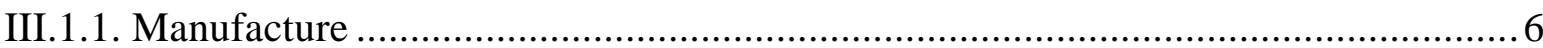

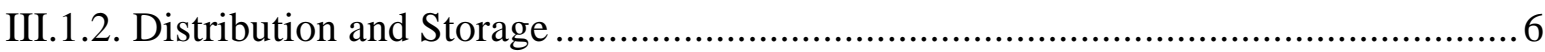

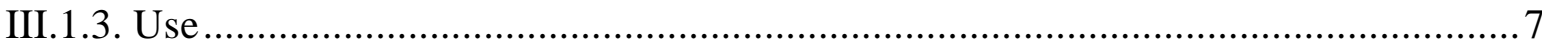

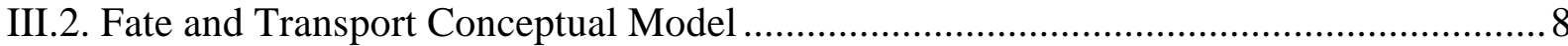

III.2.1. Limitations of the Environmental Partitioning Assessment Using the Multimedia Fugacity Approach ......................................................................................

III.2.2. Discrepancies Between Empirical Partitioning Data of PuriNOx Mixtures and Fugacity Predictions ............................................................................................ 10

III.2.3. Considerations for the Comparison of PuriNOx Additives to MTBE ......................11

III.2.4. Representativeness of Soil Column Studies ……............................................... 12

III.2.5. Issues of Vadose Zone Transport Uncertainty ........................................................ 13

III.2.6. Capabilities for Routine Analytical Measurement of Environmental

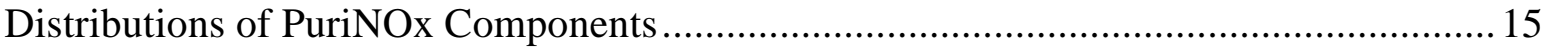

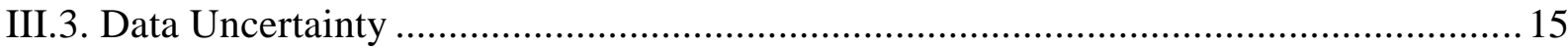

III.3.1. Use of Structural Analogs to Characterize Key Environmental Toxicology and Biodegradation Parameters .................................................................................... 15

III.3.2. Lack of Anaerobic Biodegradability Data for Many PuriNOx Components ........... 16

III.3.3. Lack of Knowledge About Biodegradation Pathways for Many PuriNOx

Components: The Potential for Metabolites that Could Degrade Water Quality .................. 17

III.3.4. Aquatic Toxicity Data Uncertainties ................................................................. 17

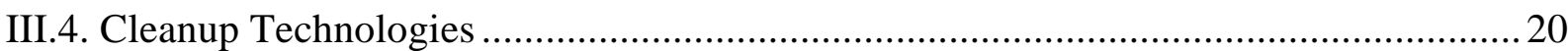

III.5. Potential for Environmental and Water Resource Impacts Relative to ULSD.................2 21

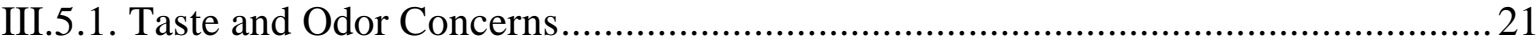

III.5.2. Concerns with Eutrophication Resulting from Releases of Component 5

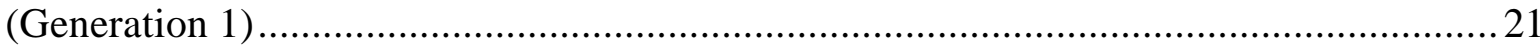

III.5.3. Possible Enhanced Solubility and Mobility of Diesel Hydrocarbons ....................... 22 
IV. Summary and Conclusions..........................................................................................................22

IV.1. Conformance of Lubrizol's Data Package to SWRCB Evaluation Criteria.....................23

IV.2. Considerations About the Fugacity Modeling Approach............................................2 23

IV.3. Discrepancies Between Empirical Partitioning Data and Fugacity Predictions............... 24

IV.4. Considerations About the Comparison of PuriNOx Components to MTBE .................. 24

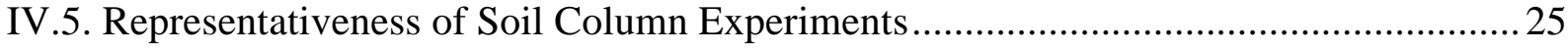

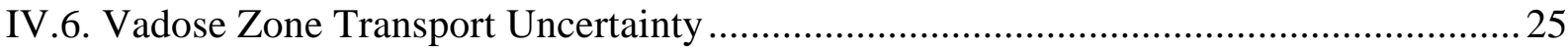

IV.7. Capabilities for Routine Analytical Measurement of PuriNOx Components .................2 25

IV.8. Use of Structural Analogs to Characterize Aquatic Toxicity and Biodegradation..........26

IV.9. Lack of Anaerobic Biodegradability Data for Many PuriNOx Components...................26

IV.10. Potential for Metabolites that Could Degrade Water Quality ……...............................2 26

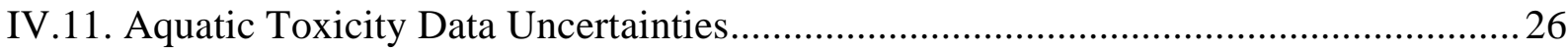

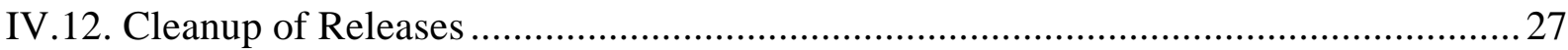

IV.13. Principal Considerations About Potential Water Resource Impacts..............................2 27

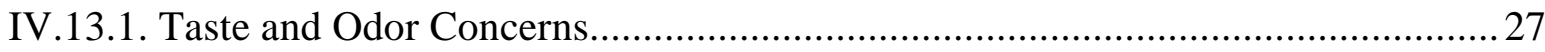

IV.13.2. Concerns with Eutrophication Resulting from Releases of Component 5

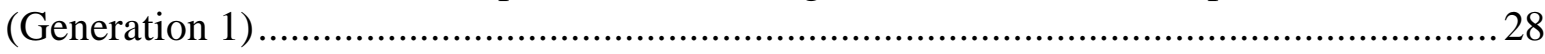

IV.13.3. Possible Enhanced Solubility and Mobility of Diesel Hydrocarbons ..................... 28

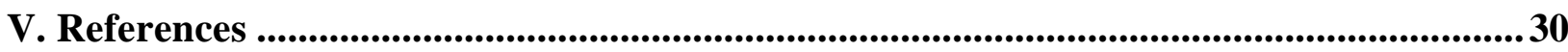

Author Biographies ............................................................................................................................33 


\section{Executive Summary and Recommendations}

\section{Introduction}

California Senate Bill 529 requires the California Environmental Policy Council to identify and evaluate all significant beneficial and adverse impacts on the environment that may result from any fuel specification proposed or established by the California Air Resources Board (CARB); this requirement includes impacts associated with the production, use, and disposal of the compound or compounds that may be used to meet the fuel specification. Additionally, California Senate Bill 989 prohibits the CARB from adopting new fuel specifications until a "multimedia" evaluation has been performed and submitted to the California Environmental Policy Council for final review and approval.

The California State Water Resources Control Board (SWRCB) is concerned with the lifecycle impacts that any new fuels may have on, and beneficial uses of, surface water and groundwater in California. In the case of PuriNOx, the SWRCB needs information that will allow an informed decision to be made regarding the relative risk of PuriNOx to California water resources and beneficial uses as compared to ultra-low sulfur diesel (ULSD). The SWRCB has made a good-faith effort to identify all of the information that may be needed and has provided this guidance to Lubrizol.

On July 16, 2003, the Lubrizol Corporation submitted a multimedia evaluation report for the use of the PuriNOx diesel fuel technology in California. Lawrence Livermore National Laboratory (LLNL) was contracted by Lubrizol to conduct an independent review of the data and data analysis included in Lubrizol's report, and to assess how the report addressed the potential impacts to surface and groundwater that may result from the proposed use of PuriNOx fuel in California. The purpose of the independent review by LLNL is to assist the SWRCB in completing its evaluation of a multimedia assessment study of the use of PuriNOx fuel as required by the $\mathrm{CARB}$ verification procedure for in-use strategies to control emissions for diesel engines.

To conduct this review, LLNL formed an independent expert panel to perform third-party review of the PuriNOx data package provided by Lubrizol. Using best professional judgment, the LLNL panel has prepared findings regarding the degree to which Lubrizol has met the SWRCB request for information concerning acceptance of PuriNOx in California, including an assessment of the completeness of the list of potential release scenarios and the fate-andtransport conceptual model proposed by Lubrizol. The panel's conclusions and recommendations are presented in this report, which has been submitted to the SWRCB and Lubrizol concurrently.

During the preparation of this report, proprietary information has been protected. The California agencies responsible for conducting the multimedia evaluation, as well as LLNL, have all signed agreements to protect this information. In many cases, specific chemicals are not named, but are referred to by designated component names that are known to the evaluation participants and reviewers.

The PuriNOx additive packages (Generations 1 and 2) are complex chemical mixtures comprising compounds ranging broadly in molecular weight from $<50$ to $>3000 \mathrm{amu}$, and ranging in aqueous solubility from the $\mu \mathrm{g} / \mathrm{L}$ range to complete miscibility with water. From both 
fuel chemistry and environmental perspectives, an important aspect of the additive packages is the inclusion of surface active compounds (emulsifiers and dispersants) that facilitate the formation of stable emulsions between water and diesel hydrocarbons.

\section{Summary and Conclusions}

\section{Conformance of Lubrizol's Data Package to SWRCB Evaluation Criteria}

Lubrizol has taken the precedent-setting challenge of being the first company to bring a new fuel to market in California under the Senate Bill 989 requirements for multimedia assessment. In this capacity, Lubrizol has made a significant good-faith effort to provide appropriate data to meet these requirements. This is a very complex challenge that has been undertaken with minimal guidance from those agencies with regulatory oversight responsibilities. The PuriNOx Fuel Multimedia Evaluation Final Report and Supplemental Information addressed all the major elements in the SWRCB data package guidance.

A further burden is the lack of general environmental information on many of the components in the PuriNOx additive package, in spite of the widespread industry use of most of these compounds. Lubrizol has provided considerable physical-chemical and toxicological data on additive components and/or structurally-related analogs, however, all of the toxicological and environmental behavior information needed to conduct a thorough evaluation is not available. Nevertheless, these constraints do not diminish any concerns that have been raised during this review; many of the criticisms and comments herein are aimed at improving the data submittals for future fuel multimedia assessments and fully informing decision makers regarding knowledge gaps.

\section{Environmental Release Scenarios}

Based on a projected market share of 40 million gallons of PuriNOx per year (by the year 2012), the volume of this additive package moving into the state will be on the order of 0.8 to 1.2 million gallons per year. This is a relatively minor amount compared to the volume of chemicals with more severe environmental consequences that are routinely transported in this manner. Although there is a potential for a release of the bulk additive package components during transport into California, the likelihood of a bulk additive package component release during transport is relatively small.

The most likely release scenarios for blended PuriNOx fuel during distribution and storage are very similar to those for ULSD. Over the long term, release of PuriNOx fuel from leaking USTs (underground storage tanks) poses the most likely release scenario; thus, an understanding of the differences in behavior between a ULSD subsurface release and a PuriNOx fuel subsurface release will be key to effective management of this new fuel. An important consideration for a release of blended PuriNOx fuel from a UST is the impact of the additive package on the fate and transport of the diesel hydrocarbons in the fuel or already present in soil as a result of previous releases or routine fueling operations.

The initial use of PuriNOx fuel in California will be centrally-fueled mobile and stationary applications and will be limited to several pilot areas. Examples of these applications and pilot areas include solid waste collection and fuel tanker trucks and the ports of Los Angeles and Long 
Beach. Since one of the highest volume deployments of PuriNOx fuel will be for port operations, there may be a release scenario in which blended fuel is released to the surface of marine waters, either through surface runoff or through a bulk spill. The impact of the additive package on the behavior of diesel fuel in a marine environment, as well as potential interactions between saltwater and additive package components, are important considerations.

\section{Considerations About the Fugacity Modeling Approach}

The Lubrizol multimedia evaluation includes a steady-state, fugacity-based simulation of the distribution of PuriNOx additive components in a landscape consisting of air, water, soil, and sediment compartments. The primary value of this multimedia simulation approach is that it provides useful diagnostic information on the likely distribution of a compound in different media as a result of alternative release scenarios, environmental properties, and various transport and fate processes.

There are limitations to the compartmental modeling approach, especially when dealing with a broad range of potential release conditions and fuel mixtures with complex chemistry. In particular, fugacity-based multimedia modeling is only applicable to dilute concentrations of a given compound in a completely mixed, homogeneous environmental compartment. Moreover, such modeling does not deal with chemical interactions between a contaminant and host media. At present, there are no methods available to make quantitative predictions of the relevant environmental properties of all surfactant classes, and of the complex interactions between surfactants and hydrocarbon fuel components in a multimedia environment.

\section{Discrepancies Between Empirical Partitioning Data and Fugacity Predictions}

Although fugacity-based models may provide a semi-quantitative idea of the partitioning of individual PuriNOx components in the environment, empirical studies provide more reliable (although not necessarily quantitative) indicators of complex partitioning behavior. The Lubrizol report presents data for the partitioning of PuriNOx and PuriNOx fuel components between water and a complex non-aqueous phase liquid (NAPL). Overall, the empirical results suggest that fugacity models may be less than reliable even in systems that are far less complex than the natural environment. For example, in a study in which PuriNOx additive packages (Generations 1 and 2) were added to water and allowed to equilibrate, Component 3 (Gen. 1) was present in water at over 700,000 times its estimated water solubility. Although the explanation may be that this compound was "dispersed," not truly dissolved, in the aqueous phase, this distinction is of dubious importance from the applied environmental perspective - both dissolved and dispersed fractions of a compound will be transported in the aqueous phase and can affect organisms in contact with that phase. The fugacity modeling presented by Lubrizol did not account for dispersion of compounds into the aqueous phase, only dissolution.

Empirical results were also difficult to explain in partitioning experiments designed to answer the question: will diesel hydrocarbons, such as BTEX (benzene, toluene, ethylbenzene, and xylenes), have enhanced water solubility after releases of PuriNOx fuel relative to ULSD? In these experiments, TPH (total petroleum hydrocarbons) and BTEX had opposite behaviors. TPH concentrations in water were 7 to 22 times higher for PuriNOx fuels than for ULSD; however, BTEX concentrations were typically 2 to 3 times lower for PuriNOx fuels than for ULSD. It seems highly implausible that surfactants would enhance the solubility of one class of 
hydrocarbons while reducing the solubility of another class. Lubrizol suggests that the confounding behavior could be an analytical artifact, since TPH and BTEX were measured by different methods that may have been variously affected by dispersed emulsion droplets. Regardless, it is questionable whether these data are sufficiently reliable to include in a conceptual transport and fate model.

\section{Considerations About the Comparison of PuriNOx Components to MTBE}

The Lubrizol report stressed the point that "the PuriNOx additive package components will not adversely impact the environment like MTBE". The predominant concept underlying the favorable comparison of PuriNOx components to MTBE is that many PuriNOx components have low water solubilities and will thus be effectively immobile in the subsurface environment. While this is a valid point, several points are noteworthy with respect to water solubility and PuriNOx components: (a) for releases to surface water, low solubility cannot necessarily be equated with low environmental concern, as insoluble, sediment-associated compounds may be ingested by benthic organisms and bioaccumulate, (b) there are PuriNOx components or hydrolysis products that are somewhat soluble that may persist in the subsurface, and (c) in addition to considering PuriNOx components themselves, we must also consider the potential effects of PuriNOx components on enhancing the solubility of toxic ULSD compounds (such as naphthalene or BTEX).

\section{Representativeness of Soil Column Experiments}

The mobility study of hydrocarbon components in soil columns undertaken by Lubrizol, in our opinion, represented the best opportunity to compare the behavior of PuriNOx fuel against that of ordinary ULSD within a multimedia context short of an actual field experiment. The soil column studies conducted by an independent laboratory on behalf of Lubrizol followed standardized protocols designed to characterize pesticide transport. Unfortunately, the experiments were not representative of likely release scenarios and critical data were not measured. Specific problems with the soil column experiments include the following: (a) the soil columns (10-mL pipettes filled with test soils to a depth of $3 \mathrm{~cm}$ ) were far too small to exclude possible edge effects exerted by the walls of the pipettes on solute transport, (b) the application of very small quantities of fuel $(100 \mu \mathrm{L})$ to the columns arguably did not emulate likely release scenarios, (c) the list of selected analytes excluded BTEX compounds, which are likely to present the issue of greatest environmental concern from a water quality perspective, and (d) the soil columns were not characterized hydraulically. Furthermore, the available data for naphthalene behavior in ULSD versus the two PuriNOx fuels (Gen. 1 and 2) suggests that naphthalene indeed was transported to a greater degree in the PuriNOx fuel releases than in ULSD releases (Section III.2.4.). While this result may not have been statistically significant, it is certainly noteworthy and contrary to the conclusion stated by Lubrizol: "The above provides conclusive evidence that mobility and transport of PuriNOx fuel in soil will be similar to ULSD."

\section{Vadose Zone Transport Uncertainty}

As with other commercial fuel products, PuriNOx diesel fuel will, in many instances, likely be released into the subsurface environment via leaking underground and above-ground fuel tanks or through cumulative spillage. As such, a number of questions emerge about the behavior 
of the PuriNOx fuel mixture, as compared to that of ordinary diesel, in unsaturated soils in the event of such a release. Examples of questions that remain, in our judgment, still outstanding include the following: (a) will surfactant compounds included in the additive package lead to the mobilization of residual hydrocarbon components entrapped in the vadose zone from prior releases, and (b) what is the fate of hydrocarbon compounds that are dissolved within water droplets emulsified within the PuriNOx fuel mixture?

\section{Capabilities for Routine Analytical Measurement of PuriNOx Components}

Conceptual transport and fate models provide a framework for predicting contaminant distributions after releases, but actual measurements must be made to characterize contamination on a site-specific basis. Notably, most PuriNOx additive components are not compounds that are routinely analyzed by commercial analytical laboratories and in fact many are not amenable to standardized analytical methods developed by U.S. Environmental Protection Agency and other agencies. Specifically, many components and their hydrolysis products are too nonvolatile (due to polar functional groups and/or relatively high molecular weight) to be analyzed by gas chromatography techniques. Therefore, in the event of a significant release, analysis for most components would require a custom analysis, typically requiring expensive analytical equipment (such as a liquid chromatograph/mass spectrometer capable of tandem mass spectrometry) that is rarely available in commercial environmental laboratories. It should be noted that many of the PuriNOx components have been in commercial use for 20 years or more, yet there are no regulatory monitoring requirements to assess their environmental release from these other uses.

\section{Use of Structural Analogs to Characterize Aquatic Toxicity and Biodegradation}

For a few PuriNOx components, structural analogs were used rather than the actual compounds to generate aquatic toxicity and aerobic biodegradation data. In general, the choice of analogs appears reasonable. However, some caution is warranted. In fact, certain biodegradation data presented by Lubrizol suggest a marked effect on degradation of a seemingly minor structural difference (Section III.3.1).

\section{Lack of Anaerobic Biodegradability Data for Many PuriNOx Components}

In most cases, assessments of biodegradation were based on the modified Sturm test, a standard assay that measures aerobic biodegradation of test substances by sewage sludge microorganisms. Although this assay may be an acceptable qualitative indicator of biodegradability under aerobic conditions, it has no relevance to anaerobic conditions, which are likely for some common release scenarios, namely, release into the subsurface via leaking USTs or release into low-energy sediment environments (such as marinas). Furthermore, anaerobic degradation rates are typically much slower than aerobic degradation rates. Thus, the concern is that the method used to generate most of the biodegradation data presented by Lubrizol may significantly overestimate biodegradability in the environment under relevant release scenarios.

\section{Potential for Metabolites that Could Degrade Water Quality}

Slow biodegradation observed for some PuriNOx components raises the question of whether problematic metabolites are accumulating during slow degradation, and whether a portion of a parent compound might be resistant to degradation, leading to the formation of persistent 
metabolic by-products. Based on consideration of the structures of slowly degraded PuriNOx components, it is not obvious that anticipated degradation pathways would result in persistent metabolites that are more problematic than the parent compounds.

\section{Aquatic Toxicity Data Uncertainties}

Comprehensive aquatic toxicity testing of the PuriNOx additive package mixtures would have been challenging. Given a choice, it would have been preferable to have aquatic toxicity data on the mixtures rather than on the individual components. However, if carefully conducted, the results from such tests would have yielded data of more immediate relevance than data on the individual compounds, as it is exposure to the mixture that will be sustained by organisms in the event of an accidental release. Among the concerns about the toxicity data are the following: (a) there is no explicit evaluation of sediment toxicity that may occur from a release of either the 'neat' additive package, or of PuriNOx fuel, despite the clear and acknowledged tendency of many of the individual additive components to partition to and persist in soil and aquatic sediments, (b) an acute exposure period with lethality as an endpoint was the only scenario considered, (c) acute aquatic toxicity criteria such as LC50 and/or EC50 values in the Lubrizol report are often well above the stated solubility of the compound, (d) from data presented, there is no basis for concluding that the PuriNOx components will not impact the endocrine system, and (e) Lubrizol equates limited water solubility to minimal or no toxicity; this relationship is not necessarily correct, and it was inappropriate to invoke limited solubility as a reason for not providing toxicity data, or to support assumptions that it is reasonable to provide aquatic toxicity data from a more soluble analog because of its presumed greater toxicity.

\section{Cleanup of Releases}

At some point, releases of PuriNOx fuel to both surface waters and the subsurface environment must be anticipated. We believe that cleanup responses to releases of the (fully mixed) fuel product to the subsurface will likely entail the application of the same remediation approaches that would normally be applied to a ULSD spill. The release of PuriNOx fuel to surface waters, particularly marine waters, may present a more difficult problem. In oil spills that have occurred in marine surface waters, water-in-oil emulsions consisting of 50 to 80 percent water form a grease-like, reddish-brown material frequently referred to as "mousse." Additionally, oil-in-water surfactants reduce the water surface tension, which can result in increased hydrocarbon movement into the water column. If mousse formation should occur, or if diesel fuel and additive components move downward into the water column, cleanup may be more problematic. Cleanup options that include mechanical containment and collection may not be practicable in either of these situations.

\section{Principal Considerations About Potential Water Resource Impacts}

Taste and Odor Concerns. As reported by Lubrizol, zero aerobic or anaerobic degradation was observed in 28 days for Component 4 (Gen. 1), which is also included in Gen. 2. Although this compound does not appear to be highly toxic, it does have a distinctive odor and appreciable water solubility (between 12 and $18 \mathrm{mg} / \mathrm{L}$ ). Therefore, it is possible that this compound may persist in water after release in the environment (particularly in the subsurface, since it may tend to volatilize from surface water). Notably, Lubrizol indicates that this compound will hydrolyze in water (half-life of 7 days at $\mathrm{pH}$ 7). If so, this would mitigate concerns about persistence. 
However, it is difficult to reconcile the lack of aerobic biodegradation with relatively rapid hydrolysis at neutral $\mathrm{pH}$, as the expected organic hydrolysis product should be relatively easy to degrade under aerobic conditions.

This biologically-recalcitrant component is commonly used as a cetane improver in diesel fuels, although the concentration added to PuriNOx fuel is more than an order of magnitude higher than that added to ULSD. With regard to Component 4 (Gen. 1), Lubrizol has provided the following information: "A test plan is being prepared by the American Chemistry Council (ACC) Lubricant Additives Industry Consortium under the U.S. EPA High Production Volume (HPV) Chemical Challenge program. This will enable a complete environmental dossier to be developed based on physical-chemical and toxicity testing on this chemical which will be publicly available on the EPA HPV web site."

There are also potential taste/odor concerns with the two substituted alkanolamine components or hydrolysis products associated with Components 1 and 3 (Gen. 1) and Components 1 and 2 (Gen. 2). These compounds have relatively high water solubility and a distinctive, fishy odor. Although both compounds are apparently degradable under aerobic conditions, there is no information on their anaerobic biodegradability.

Concerns with Eutrophication Resulting from Releases of Component 5 (Generation 1). One concern with environmental releases of PuriNOx additive package or PuriNOx fuels is that the two constituents of Component 5 (Gen. 1), which is also included in Gen. 2, will promote eutrophication in surface waters. These constituents are highly soluble in water and are known to serve as fertilizers that can promote algal blooms. Whether eutrophication will result from PuriNOx releases will depend in large part on the potential for dilution in the affected surface water body.

Since the anionic constituent of Component 5 is regulated in groundwater, subsurface releases could also be a concern. However, if the subsurface release is of PuriNOx fuel, it is likely that this compound will serve as an electron acceptor for anaerobic hydrocarbon degradation and will not persist.

Possible Enhanced Solubility and Mobility of Diesel Hydrocarbons. Considering the surfactant properties of many PuriNOx components, an obvious concern about releases of PuriNOx fuels is that aqueous concentrations of diesel hydrocarbons (naphthalene and BTEX, in particular) could be enhanced relative to the concentrations resulting from releases of ULSD. As discussed in Sections III.2.1, III.2.4, and III.2.5, PuriNOx components could facilitate mobilization of residual hydrocarbons entrapped in the vadose zone and could also enhance the solubilization of hydrocarbons in the saturated zone. Existing fugacity models cannot address these issues, and the empirical partitioning and soil column experiments reported by Lubrizol do not resolve them, because of limitations in experimental design and/or equivocal results (Sections III.2.1, III.2.2, III.2.4, and III.2.5). Thus, questions about enhanced solubility and mobility of diesel hydrocarbons after releases of PuriNOx fuel remain unresolved.

\section{Recommendations to Address Key Uncertainties}

The limited initial use of PuriNOx fuel will tend to constrain the number of potential release sites. Given the uncertainties associated with the complexities of the additive package chemistry and with site-specific variables, these limited applications provide an important opportunity for 
extra vigilance to gain practical environmental experience with this potentially beneficial new fuel. In the event of a release, characterization efforts should consider the issues raised in this report. A key element to using this approach will be improvements in analytical chemistry capabilities that will enable effective monitoring of PuriNOx components.

\section{Assessment of Actual Environmental Distributions After a Known Release of PuriNOx Additive or PuriNOx Fuel}

As described in this report, there are inherent limitations in the use of fugacity models and empirical laboratory data to accurately predict chemical distributions in the natural environment. The best way to reliably assess environmental distributions is to measure them directly after a release. As some releases of transportation fuels are inevitable, it would be beneficial to use a known release of PuriNOx additive or fuel as a learning opportunity. The list of compounds analyzed in water should include not only those with relatively high water solubilities, but also the less soluble compounds that may be dispersed in water (e.g., see Section III.2.2). Special attention should be given to compounds highlighted in Section III.5. Since analytical methods are not available for certain PuriNOx components, methods may have to be developed for those compounds (see next section). In the event of a PuriNOx fuel release, behavior of regulated hydrocarbons (e.g., BTEX and naphthalene) should be assessed. For well-characterized, contaminated aquifer sites with historical hydrocarbon data, it may be possible to determine whether BTEX concentrations have increased as a result of the presence of surfactants in PuriNOx fuel.

\section{Development of Analytical Methods for PuriNOx Components of Greatest Concern}

As noted in the previous section, direct measurements of PuriNOx components after a known release would be an opportunity to learn more about their transport and fate. PuriNOx components, particularly those of greater concern (e.g., those highlighted in Section III.5.1), can be analyzed by gas chromatography/mass spectrometry or liquid chromatography/mass spectrometry (or preferably, tandem mass spectrometry) with electrospray ionization. As indicated in the Lubrizol report, analyses of these compounds should be feasible. However, challenges will exist, including (a) rigorous quantification, which will rely on authentic standards and stable isotope-labeled internal standards that are not commercially available (a particular problem exists for PuriNOx components that are actually a mixture of compounds, such as Component 1, Gen. 1) and (b) disruption of the electrospray ionization mechanism in the presence of high concentrations of surface active compounds, which will place greater significance on the use of internal standards.

\section{Biodegradation Studies to Fill Important Data Gaps}

As noted in Section III.3.2, there is no information on the anaerobic biodegradability of some PuriNOx components, although some release scenarios would tend to occur under anaerobic conditions (such as leaking USTs). We recommend that studies be undertaken to assess the degradation of these components under a range of electron-accepting conditions that can be expected in the environment (i.e., denitrifying, sulfate-reducing, ferric iron-reducing, and methanogenic). 
In particular, the biodegradation of Component 4 (Gen. 1) merits further study, as this compound was reported to undergo zero degradation in 28 days under aerobic and anaerobic conditions. More detailed assessment of this compound's degradability would be useful to assess its long-term fate.

For the PuriNOx components that were observed to degrade slowly under aerobic conditions (or those subsequently found to degrade slowly under anaerobic conditions), more detailed studies could be useful to identify whether problematic metabolites are accumulating (as discussed in Section III.3.3). These studies would require the measurement of metabolites in culture medium rather than the modified Sturm test, which only measures mineralization to $\mathrm{CO}_{2}$. Therefore, more sophisticated analytical procedures would be required for these studies.

\section{Aquatic Toxicity Testing}

While leakage from USTs was identified as the most likely release scenario over the longer term, there remains a potential for releases to surface waters. As part of longer-range studies to fill important data gaps, we recommend that studies be conducted to evaluate the chronic toxicity of the PuriNOx additive packages - as a mixture, not as the individual components - to a range of representative aquatic organisms. We recommend that such studies be designed to evaluate effects on reproduction, development, behavior, and various indicators of systemic toxicity. We also recommend that research be conducted to delineate both acute and chronic effects of the PuriNOx additive package (as a mixture, not individual components) on sediment organisms. Depending on the results of those studies, additional consideration of food-chain effects and/or bioaccumulation may also be warranted.

\section{Soil Column Studies}

Soil column studies provide the most direct means, aside from a field-scale study, for assessing the potential for fuel mixture components to migrate through the subsurface. As discussed in Section III.2.4, there were a number of shortcomings identified in the initial comparative column studies of PuriNOx fuel and ULSD. In studies that may be conducted as part of an evaluation of other fuel mixtures in the future, we recommend that experimental soil columns be of sufficient size to allow for proper hydraulic characterization and control, that conservative tracers be used to quantify a baseline breakthrough curve, that retardation coefficients be calculated for fuel components and additives, and that all important constituents of interest be analyzed (including BTEX - this last issue also applies to comparative biodegradation studies). To the extent possible, attempts should be made to emulate likely release scenarios. In the interim, because the soil column studies that were conducted provided very limited information, we recommend that sites where subsurface releases of PuriNOx are known or suspected be carefully monitored to ascertain the extent of transport of diesel hydrocarbons and additive package constituents in the subsurface. 


\section{Introduction}

\section{II.1. Background}

\section{II.1.1. California Multimedia Law}

California Senate Bill 529 requires the California Environmental Policy Council, which was established pursuant to Section 71017 of the Public Resources Code, to identify and evaluate all significant beneficial and adverse impacts on the environment that may result from any fuel specification proposed or established by the California Air Resources Board (CARB), including impacts associated with the production, use, and disposal of the compound or compounds that may be used to meet the specification. Additionally, California Senate Bill 989 prohibits the CARB from adopting new fuel specifications until a "multimedia" evaluation has been performed and submitted to the California Environmental Policy Council for final review and approval. Further, the requirement for a multimedia evaluation is noted in the California Air Resources Board Proposed Regulation for the Verification Procedure for In-Use Strategies to Control Emissions from Diesel Engines.

The evaluation that the Council is required to prepare must, at a minimum, address impacts associated with all of the following:

- Emissions of air pollutants, including ozone-forming compounds, particulate matter, toxic-air contaminants, and greenhouse gases

- Contamination of surface water, groundwater, and soil

- Disposal of waste materials, including agricultural residue, forest biomass, and municipal solid waste.

If the Council determines that the overall impact on the environment is adverse, or that alternatives exist that would be less adverse, the Council shall recommend alternative specifications or other measures that the state board or other state agencies may take to reduce the adverse environmental impacts.

\section{II.1.2. SWRCB Evaluation Criteria}

The California State Water Resources Control Board (SWRCB) is concerned with the lifecycle impacts that any new fuels may have on, and beneficial uses of, surface and groundwater in California. These beneficial uses include, but are not limited to, the following: municipal and domestic supply, water contact recreation, and freshwater/marine/estuarine habitat and wildlife habitat. Protection of these uses addresses a broad range of considerations ranging from effects on human health, to nuisance (e.g., taste and odor), to fish toxicity.

In the case of PuriNOx, the SWRCB needs information that will allow an informed decision to be made regarding the relative risk of PuriNOx to California water resources and beneficial uses as compared to ultra-low sulfur diesel (ULSD). In addition, the SWRCB needs information specifically related to the potential risk of the additive package to resources and uses insomuch as it is transported and stored separately. The SWRCB has made a good faith effort to identify all 
of the information that may be needed and provided this guidance as an appendix to a Letter of Understanding Regarding Providing Independent Review of PuriNOx Data, dated September 30, 2002.

As specified by the SWRCB, the data package submitted by Lubrizol should contain the following components.

1. Environmental Release Scenarios. The description of the potential environmental release scenarios of PuriNOx fuel and the additive package should entail a life cycle perspective, including an evaluation of which scenarios pose the greatest threat to human health, the environment, and beneficial use of water resources. This evaluation should also include an estimation of the likelihood of occurrence for each scenario and the basis for that estimate.

Background information should address the following issues:

- What is the specific make-up of the additive package and PuriNOx fuel?

- How will the additive package and PuriNOx fuel be manufactured, transported, and stored?

- What is the history of use/ other uses?

- Is there any history of water quality problems associated with the discharge of any component of the new fuel?

Possible release scenarios that should be considered include the following:

- Catastrophic release of PuriNOx fuel or the additive package during rail or truck transport into California. Releases to both freshwater and marine environments should be considered.

- Release of PuriNOx fuel or additive package from a bulk storage container at a mixing facility.

- Slow release of PuriNOx fuel or additive package from an underground storage tank.

- Catastrophic release of PuriNOx fuel or additive package from an underground storage tank.

2. Fate and Transport Conceptual Model. A fate and transport conceptual model is defined as a set of hypotheses or assumptions regarding the behavior of fuel components in the environment. A description of the fate and transport conceptual model for releases of PuriNOx fuel into both surface and subsurface waters should emphasize differences relative to releases of ULSD. There should also be consideration of fuel transport as a non-aqueous phase liquid (NAPL) and as a vapor phase. In the subsurface, this should include consideration of the processes that occur under saturated and unsaturated groundwater conditions and should consider the interaction of the fuel with the soil matrix. Justification, literature support, and discussion of the basis of the conceptual model assumptions and hypotheses should be provided. Typically the description of the fate and transport conceptual model should include basic physical and chemical properties of the new fuel components and the finished fuel, such as: 
- Solubility in water

- Volatility

- Specific gravity

- Corrosivity (especially to storage containers)

- Permeability

- $K_{\mathrm{OC}}$

- Henry's Law coefficient.

Fate and transport conceptual model questions that should be addressed include the following:

- Will there be any changes in tailpipe emissions that could affect water quality (i.e., through washout)?

- What are the effects on capillary and soil pore conditions and partitioning within the soil environment?

- What are the effects on the fate and transport of surface and groundwater plumes? Once it reaches water, will a PuriNOx fuel plume move faster or farther or be more persistent than ULSD?

- Will there be any relative change in biodegradation rates?

- What will be the ultimate fate of the product by component as compared to ULSD or for the new components in PuriNOx fuel that are not already in diesel (mass balance)?

- Will daughter products be generated during natural environmental transformation processes and what is the hazard associated with these daughter products?

- What will be the impact if a release commingles with existing soil/groundwater contaminated with petroleum hydrocarbons or MTBE? Specifically, will PuriNOx mobilize petroleum contaminants in soil or groundwater?

3. Data Uncertainty. Uncertainty in the current state of knowledge regarding PuriNOx should be discussed throughout the data package and key uncertainties should be identified. If experimental data are provided, standards, tests, and experiments used to generate these data must be fully described, and discussed along with proper experimental controls. Whenever possible, standardized methods should be employed.

4. Summary of the Regulatory Approval Status. This should include any individual state or national regulatory approvals that are available or in progress and any governmentadopted health criteria.

5. Screening Risk Analysis. A screening risk analysis for potential environmental, and resource impacts that may result from the identified most hazardous and/or likely release scenarios. This risk analysis typically should address issues such as: 
- Results of aquatic toxicity tests, including effects on benthic organisms, fish and plants

- Taste and odor characteristics in drinking water

- Effects on color/clarity of water.

6. Cleanup Technologies. How would a release of PuriNOx respond to standard petroleum cleanup technologies and strategies? Would the fact that the diesel is already emulsified reduce the ability to contain and clean up a release to surface water? Would PuriNOx fuel be easier or more difficult to clean up?

\section{II.1.3. The LLNL Expert Panel's Role}

On July 16, 2003, the Lubrizol Corporation submitted to the SWRCB and CARB a multimedia evaluation report for the use of the PuriNOx diesel fuel technology in California. Lawrence Livermore National Laboratory (LLNL) was contracted by Lubrizol to conduct an independent review of data and data analysis developed by Lubrizol regarding the potential impacts to surface and groundwater that may result from the proposed use of PuriNOx fuel in California. The purpose of the independent review by LLNL is to assist the SWRCB in completing its evaluation of a multimedia assessment study of the use of PuriNOx fuel as required by the CARB verification procedure for in-use strategies to control emissions for diesel engines (See Letter of Understanding Regarding Providing Independent Review of PuriNOx Data, dated September 30, 2002). However, the SWRCB reserves the right to request additional information and to arrive at conclusions that may differ from those of the LLNL panel (see Appendix A, Letter of Understanding Regarding Providing Independent Review of PuriNOx Data).

To conduct this review, LLNL formed an independent expert panel to perform third-party review of the PuriNOx data package provided by Lubrizol. Although this effort is supported through a contractual agreement between LLNL and Lubrizol, LLNL conducted an independent evaluation of data provided by Lubrizol and submitted work products directly to the SWRCB and Lubrizol. The panel's conclusions and recommendations are presented in this report, which has been sent to the SWRCB and Lubrizol concurrently.

Using best professional judgment, the LLNL panel has prepared findings regarding the degree to which Lubrizol has met the SWRCB request for information concerning acceptance of PuriNOx in California, including an assessment of the completeness of the list of potential release scenarios and the fate and transport conceptual model proposed or developed by Lubrizol. The expert panel has also included findings on potential environmental and resource impacts that may occur if PuriNOx fuel is used in California, identified important data or knowledge gaps, and recommended steps or actions to address these uncertainties.

During the preparation of this report, proprietary information has been protected. The California agencies responsible for conducting the multimedia evaluation, as well as LLNL, have all signed agreements to protect this information. In many cases, specific chemicals are not named, but are referred to by designated component names that are known to the evaluation participants and reviewers. 


\section{II.2. Summary of the Regulatory Approval Status for PuriNOx}

A summary of regulatory approvals that Lubrizol has obtained for PuriNOx is provided in Lubrizol's report and Supplementary Information. While these approvals and verifications by the U.S. EPA (Environmental Protection Agency) and CARB indicate the air benefits of PuriNOx, these regulatory evaluations focused primarily on emissions and exhaust components. The potential multimedia impacts are the subject of the present Lubrizol data submittal and the LLNL review.

It should be noted that many of the chemicals used as part of the PuriNOx additive package have been in use in the transportation marketplace at significant quantities and for extended periods of time. Though they have been scrutinized under chemical control laws in the U.S. and various regions of the world as part of new chemical notification processes, this will represent the first time many of these chemicals have undergone scrutiny for multimedia impacts during use in a transportation fuel. Lubrizol has provided considerable physical-chemical and toxicological data on additive components and/or structurally-related analogs, however all of the toxicological and environmental behavior information needed to conduct a thorough evaluation is not available. 


\section{Review of Submitted Data and Identification of Critical Knowledge Gaps}

\section{III.1. Environmental Release Scenarios}

The potential release scenarios presented by Lubrizol for the PuriNOx additive package and blended fuel focus exclusively on various kinds of spills during blending, distribution, and storage. Releases during manufacture of the PuriNOx additive package were not considered, as the PuriNOx additive package is produced outside of California. From a comparative standpoint, the primary differences between the potential release mechanisms of a CARB ULSD and PuriNOx fuel are the releases that could occur during (1) the transport of the additive package to a blending site, (2) storage of the additive package prior to blending, (3) blending operations, and (4) releases during the storage and use of blended fuel. As presented in Lubrizol's Multimedia Evaluation (p. 89), spills and leaks of the additive package could impact surface soil, surface water, and groundwater. Aquatic sediments were not identified as an environmental medium of concern, although both deposited and suspended sediments were evaluated as part of the fugacity modeling and were predicted to be significant sinks for certain PuriNOx components when released to water.

\section{III.1.1. Manufacture}

During the deployment into the California market, blending of the PuriNOx additive package with ULSD is to be accomplished at a major refinery or major fuel distribution site initially using either a 5-million or a 25-million gallon-per-year blending unit. The potential failure modes during these step-wise blending processes are not detailed, but since the blending of the PuriNOx fuel is intended to occur within the boundaries of an operating refinery, spill management controls and capabilities at these facilities will likely be adequate.

\section{III.1.2. Distribution and Storage}

Bulk additive used to blend PuriNOx will be manufactured in Ohio and transported via rail tanker car or tanker truck to a California PuriNOx fuel blending site, which will typically be located at a major refinery or major fuel distribution site. Based on a projected market share of 40 million gallons of PuriNOx per year (by the year 2012), the volume of this additive package moving into the state will be on the order of 0.8 to 1.2 million gallons per year. This is a relatively minor amount compared to the volume of chemicals with more severe environmental consequences that are routinely transported in this manner. While there is a potential for a release of the bulk additive package components during transport into California, the likelihood of a bulk additive package component release during transport is relatively small.

Once blended with ULSD, the PuriNOx fuel will be transported, typically by tanker truck, to point-of-use storage tanks. The initial use of PuriNOx fuel in California will be centrally-fueled mobile and stationary applications and will be limited to several pilot areas. The PuriNOx technology will be distributed under the name Proformix by the ChevronTexaco Corporation and under the name PuriNOx by authorized fuel distributors. The storage of PuriNOx blended fuel could be in above-ground storage tanks (ChevronTexaco 2003) as well as underground storage 
tanks (USTs). If the use of PuriNOx-based fuels becomes more widespread, then USTs may become more widely used and subsurface releases would be an important concern.

Pilot use areas include the ports of Los Angeles and Long Beach, and since this will be the highest volume deployment and will be related to port operations, there may be a release scenario in which blended fuel is released to the surface of marine waters, either through surface runoff or through a bulk fuel spill. The impact of the additive package on the behavior of diesel fuel in a marine environment, as well as potential interactions between saltwater and additive package components, are important considerations.

Over the longer term, it is the release of PuriNOx from leaking USTs that poses the most likely release scenario; thus, an understanding of the differences in behavior between a ULSD subsurface release and a PuriNOx fuel subsurface release will be key to effective management of this new fuel. An important consideration for a release of blended PuriNOx fuel from a UST is the impact of the additive package on the fate and transport of the diesel hydrocarbons in the fuel or already present in soil as a result of previous releases or routine fueling operations. We devote a major portion of this review to the adequacy of the information provided by Lubrizol to support an understanding of these issues.

Additionally, there are some minor differences in operating a UST containing PuriNOx fuel compared to one containing ULSD. A requirement for the proper storage of blended PuriNOx fuel is that tank circulation is necessary to maintain a suspended emulsion. If the blended fuel is allowed to stand without circulation, settling of the water emulsion will begin to occur. As described by Lubrizol (Supplemental Information dated 9/4/2003, pp. 4-5), settling does not mean that the water has separated from the fuel - the emulsion will still be intact. The effect of gravity over time causes the larger emulsion droplets to settle, resulting in an emulsion with somewhat higher water content at the bottom of the tank.

As is the case with tanks containing ULSD, bottom water can form through condensation, rainwater, or other naturally-occurring events associated with handling diesel fuel. With respect to the blended fuel, according to Lubrizol, an "extraordinary" event needs to occur to break the emulsion and contribute to free water at the bottom of a storage tank. Factors that may contribute to the breakup of the emulsion include chemical contamination (Supplemental Information dated 9/4/2003, pp. 4-5).

It may be anticipated that bottom water that forms will be fully saturated with any soluble (and dispersed) components of the fuel. In the event of an un-maintained tank that has a slow leak at or near the bottom, this bottom water could be the first of the tank's contents released to the subsurface. Under these circumstances, a leaking tank would initially release contaminated water to subsurface soils, and this bottom water would then migrate down toward the water table.

\section{III.1.3. Use}

Initial applications of PuriNOx fuel in California include on-road activities such as pick-up and delivery fleets, urban school busses, solid waste collection, and fuel tanker trucks. Off-road applications include agriculture, mining equipment and engines, and stationary power generation. 
Emissions testing of PuriNOx fuel along with the baseline CARB diesel fuel demonstrated that the PuriNOx fuel provides significant reductions in emissions of CO, NOx, and PM (particulate matter) compared with the baseline fuel. The reduced emissions are attributed to the incorporation of water into the fuel, which (1) produces a spray pattern that enhances combustion, (2) lowers combustion temperature, thereby reducing the NOx emissions, and (3) delays combustion to reduce the formation of particulates. Hydrocarbon emissions were higher for PuriNOx fuel than for CARB baseline fuel; however, they are at least $25 \%$ lower than any applicable diesel vehicle emission standard (CARB verification letter dated January 31, 2001). Higher hydrocarbon emissions may be due to the effect of water on combustion timing and duration. Although there were differences in the emissions of unregulated PAH/NPAH (i.e., polycyclic aromatic hydrocarbons/nitrated PAH) between the PuriNOx and baseline fuels (as expected, given changes in combustion kinetics), the total amount of PAH/NPAH compounds in vapor and particulate phases were not significantly different between the fuels tested. Moreover, the semivolatile compounds detected in the emissions were essentially identical for PuriNOx and baseline CARB fuels (methanol was the only exception because it was a constituent of the PuriNOx blend used).

It is possible that uncombusted additive components from the Generation 1 (Gen. 1) and Gen. 2 PuriNOx formulations may be present in the exhaust. To further support a comparative multimedia assessment of PuriNOx fuel and the CARB baseline diesel, additional data are needed to determine whether un-combusted additive components from Gen. 1 and Gen. 2 formulation packages exist in the emissions. Atmospheric emissions are not specifically listed in Lubrizol's conceptual release model (p. 89), yet they are simulated in the multimedia fugacity modeling effort (p. 61). The multimedia modeling predicts that soil and sediments will be important reservoirs for various constituents of both additive packages after airborne releases [e.g., Components 1 and 2 (Gen. 1)]. Given that other unregulated combustion products from PuriNOx and CARB baseline fuels could also end up in surficial soils (e.g., polycyclic combustion products), how would the predicted buildup of un-combusted additives in soil compare with levels of PAH under various emissions scenarios? Clearly, to address this question, measurements would be needed of specific additives and/or surrogate compounds during an emissions testing protocol. Once emission rate data are available, then the requisite comparisons can be made between the PuriNOx and baseline CARB diesel fuels. However, it is unlikely that standardized analytical methods could support such a mass balance emissions assessment, and therefore such data are not available to Lubrizol for diesel fuel or any fuel additives currently used.

\section{III.2. Fate and Transport Conceptual Model}

\section{III.2.1. Limitations of the Environmental Partitioning Assessment Using the Multimedia Fugacity Approach}

The transport and fate of fuels and associated additives in environmental media can be assessed in terms of their media-specific behavior (e.g., release to soil followed by transport in the soil column) as well as their multimedia fate in a given landscape (e.g., releases to air that end up in surficial soils due to rainout from the atmosphere). The Lubrizol multimedia evaluation includes a steady-state, fugacity-based simulation of the distribution of PuriNOx 
additive components in a landscape consisting of air, water, soil, and sediment compartments. The fate of a compound in the compartmental landscape is a function of intermedia transfers (e.g., mass-transfer processes such as volatilization from water to air), advection, and degradation via biotic and/or abiotic processes. The primary value of this multimedia simulation approach is that it provides useful diagnostic information on the likely distribution of a compound in different media as a result of alternative release scenarios, environmental properties, and various transport and fate processes.

There are limitations to the compartmental modeling approach, especially when dealing with a broad range of potential release conditions and fuel mixtures with complex chemistry. In particular, fugacity-based multimedia modeling is only applicable to dilute concentrations of a given compound in a completely mixed, homogeneous environmental compartment. Moreover, such modeling does not deal with chemical interactions between a contaminant and host media. For example, some of the major components of the PuriNOx Generation 1 and 2 packages are surface-active agents (surfactants). The basic structure of a surfactant consists of a polar moiety (hydrophilic group) and nonpolar one (hydrophobic tail). In the case of the PuriNOx additives, the hydrophobic tails are simply aliphatic hydrocarbons that are chemically similar to the basic components of any petroleum-based fuel. The distinctive feature that separates these major components from a regular petroleum-based fuel is the polar moiety. At present, there are no methods available to make quantitative predictions of the relevant environmental properties of all surfactant classes (Boethling and Mackay 2000).

The surfactants in question are large molecules with negligible vapor pressure, thus their fate in the environment (soil, sediment, and water) is determined mainly by biodegradation and sorption. Surfactants have the tendency to accumulate at interfaces where the hydrophobic tail can avoid interaction with water molecules while the polar group can remain hydrated. This tendency underlines the importance of sorption in their environmental fate, especially when the ratio of water-to-solid volume is small. In water, the energetically unfavorable interactions with the hydrophobic tails can cause aggregates to form. These aggregates (micelles) start forming at a critical concentration that can be defined as the molecular solubility of the surfactant. Micelles remain dispersed in the water, as electrostatic repulsion prevents them from coalescing. These micelles create hydrophobic microenvironments where compounds that are normally insoluble in water can become waterborne, increasing their mobility in aqueous systems. Regional scale fugacity modeling cannot handle such chemical processes.

A specific shortcoming of the fugacity approach exists with respect to the soil partitioning model employed by Lubrizol to estimate bulk soil concentrations for select additive package components. This equilibrium partitioning model is based on a linear adsorption isotherm, Henry's Law, and various assumptions concerning representative unsaturated soil properties. The resulting modeled bulk soil concentrations are presented by Lubrizol largely without any supporting context; the reader is left to draw his or her own conclusions as to the implications for the environmental fate of the compounds simply from the listed soil concentrations. A more informative approach would have been to integrate the partitioning model into an overall conceptual model that could include, for example, the implied chemical pulse travel velocities in soil water or groundwater following a release with respect to the rate of bulk pore water movement. Using the same list of parameters provided as input in the Lubrizol soil partitioning model, one may employ the concept of a retardation coefficient, typically a key element of a 
groundwater fate and transport model for a strongly adsorbing contaminant, to quantify the rate of contaminant plume migration:

$$
R=\frac{v}{v_{c}}=1+\frac{K_{o c} f_{o c} \rho_{b}}{\theta_{w}}
$$

where $R$ is the retardation coefficient, $v$ is the bulk pore water velocity, $v_{c}$ is the velocity of the contaminant pulse, and the remaining parameters are as defined in the Lubrizol evaluation (i.e., $K_{o c}$ the organic carbon partitioning coefficient, $f_{o c}$ the organic carbon content of the soil, $\rho_{b}$ the soil bulk density, and $\theta_{w}$ the water-filled porosity). As an example, using the chemical data and soil property assumptions provided by Lubrizol for Component 4 (Gen. 1), the implied retardation coefficient for this compound is approximately 8.9, suggesting that the compound would migrate through a soil column, or through groundwater in the saturated zone, at a rate roughly one order of magnitude less than that of bulk fluid movement. Such estimates would provide a useful context for understanding the implications of soil partitioning.

Lubrizol attempted to conduct batch soil experiments, in accordance with ASTM guidelines, to quantify partitioning of individual additive package components. However, because of the low solubility of the components as well as analytical difficulties with the sample matrix, the experiments were not successful. As an alternative means of validating the soil partitioning model, the flow-through soil column experimental apparatus that was used to perform the soil leaching tests (see Section III.2.4) could have been used to estimate retardation coefficients for additive package components via the quantification of breakthrough curves. This test was not attempted. Consequently, Lubrizol's soil partitioning model, while providing some limited insight as to the environmental fate of some additive components, is incomplete because it has not been verified, and, as such, is not fully convincing in the form presented.

\section{III.2.2. Discrepancies Between Empirical Partitioning Data of PuriNOx Mixtures and Fugacity Predictions}

Although fugacity-based models may provide a semi-quantitative idea of the partitioning of individual PuriNOx components in the environment, empirical studies provide more reliable (although not necessarily quantitative) indications of complex partitioning behavior. The Lubrizol report presents data for the partitioning of PuriNOx and PuriNOx fuel components between water and a complex NAPL. Since several components of PuriNOx are surface active and can enhance the partitioning of polar compounds (such as water) into nonpolar phases (such as diesel fuel), and vice versa, chemical behavior can be complicated and very difficult to predict (Section III.2.1).

Overall, the empirical results suggest that fugacity models may be less than reliable even in systems that are far less complex than the natural environment.

The empirical results of the partitioning studies reported by Lubrizol are problematic for some compounds. In a study in which PuriNOx additive packages (Generations 1 and 2) were added to water and allowed to equilibrate (p. 96), Component 3 (Gen. 1) was present in water at over 700,000 times its estimated water solubility. Although the explanation may be that this 
compound was "dispersed," not truly dissolved, in the aqueous phase, this distinction is of dubious importance from the applied environmental perspective - both dissolved and dispersed fractions of a compound will be transported in the aqueous phase and can affect organisms in contact with that phase. The fugacity modeling presented by Lubrizol did not account for dispersion of compounds into the aqueous phase, only dissolution. Since, for Component 3 (Gen. 1) and possibly other PuriNOx components (e.g., Component 2, Gen. 2), the dispersed fraction will be far greater than the dissolved fraction, the fugacity results could be misleading for such compounds. The empirical partitioning behavior of compounds that should be even less soluble than Component 3 (Gen. 1) could not be determined due to analytical difficulties.

In contrast to the anomalously high aqueous concentration observed for Component 3 (Gen. 1), the two ionic constituents of Component 5 (Gen. 1) were present in water at only $<0.5 \%$ of their water solubilities (and represented $\leq 52 \%$ of their total mass) after two weeks of equilibration (p. 96). Much lower-than-expected concentrations of these ionic compounds were also reported in studies with PuriNOx fuel (p. 105). [Note: Calculations by Lubrizol in the tables on p. 96 and 105 were originally in error for these compounds, as their concentrations in the additive package should have been equal on a molar basis but were listed as equal on a weight basis. The preceding text is consistent with corrections subsequently provided by Lubrizol on September 4, 2003.]

Empirical results were also difficult to explain in partitioning experiments designed to answer the question: will diesel hydrocarbons, such as BTEX (benzene, toluene, ethylbenzene, and xylenes), have enhanced water solubility after releases of PuriNOx fuel relative to ULSD? In these experiments (p. 103), partitioning of hydrocarbons into water was compared among ULSD, PuriNOx fuel (Gen. 1), and PuriNOx fuel (Gen. 2). At higher spiking concentrations, Total Petroleum Hydrocarbons (TPH) and BTEX hydrocarbons had opposite behaviors. TPH concentrations in water were 7 to 22 times higher for PuriNOx fuels than for ULSD; however, BTEX concentrations were typically 2 to 3 times lower for PuriNOx fuels than for ULSD. It seems highly implausible that surfactants would enhance the solubility of one class of hydrocarbons while reducing the solubility of another class. Lubrizol suggests that the confounding behavior could be an analytical artifact, since TPH and BTEX were measured by different methods that may have been variously affected by dispersed emulsion droplets. Regardless, it is questionable whether these data are sufficiently reliable to include in a conceptual transport and fate model.

\section{III.2.3. Considerations for the Comparison of PuriNOx Additives to MTBE}

The Lubrizol report stressed the point that "the PuriNOx additive package components will not adversely impact the environment like MTBE" (p. 7), and devoted Section 5.4 of their report to this point. The predominant concept underlying the favorable comparison of PuriNOx components to MTBE is that many PuriNOx components have low water solubilities (and accordingly, high $K_{\text {oc }}$ values) and will thus be effectively immobile in the subsurface environment. While this is a valid point, it must also be acknowledged that compounds can be different than MTBE (e.g., very sparingly soluble in water, such as benzo[a]pyrene or other $\mathrm{PAH}$ ) and still be of environmental concern. With respect to water solubility and PuriNOx components, several points are noteworthy: (a) for releases to surface water, low solubility cannot necessarily be equated with low environmental concern, as insoluble, sediment-associated 
compounds may be ingested by benthic organisms and bioaccumulate, (b) there are PuriNOx components or hydrolysis products that are somewhat soluble that may persist in the subsurface (see Section III.5.1), and (c) in addition to considering PuriNOx components themselves, we must also consider the potential effects of PuriNOx components on enhancing the solubility of toxic ULSD compounds (e.g., the potential of surfactants in PuriNOx to enhance the solubility of naphthalene or BTEX compounds; see Sections III.2.1, III.2.2, and the end of Section III.2.4).

Another important comparison of PuriNOx components to MTBE relates to mass loading from a release. As pointed out in the Lubrizol report, MTBE typically constitutes 11 vol\% of gasoline, whereas the typical additive treat rate in PuriNOx fuel is 2 to $3 \mathrm{wt} \%$. The components addressed in Section III.5.1 would constitute 0.23 to $0.71 \mathrm{wt} \%$ of PuriNOx fuel but 10 to $31 \mathrm{wt} \%$ of the additive package.

\section{III.2.4. Representativeness of Soil Column Studies}

The mobility study of hydrocarbon components in soil columns undertaken by Lubrizol, in our opinion, represented the best opportunity to compare the behavior of PuriNOx fuel against that of ordinary ULSD within a multimedia (i.e., soil + water) release context short of an actual field experiment. The soil column studies were conducted by an accredited independent laboratory on behalf of Lubrizol in accordance with U.S. EPA FIFRA (Federal Insecticide, Fungicide, and Rodenticide Act) and Good Laboratory Practices guidelines that pertain to protocols designed to study the mobility and behavior of pesticides in the environment. However, the experiments were not representative of likely release scenarios and critical data were not measured. Specific problems with the soil column experiments include the following:

- The soil columns used in the experiments (10-mL, 1-cm inner diameter Pyrex pipettes filled with test soils to a depth of $3 \mathrm{~cm}$ ) were far too small to exclude possible edge effects exerted by the walls of the pipettes on solute transport. Given the textural range in soil composition, notably the variability in clay and sand fraction contents (11\% - 43\% and 25\% - 63\%, respectively), a considerable range in soil hydraulic conductivity, and hence solute travel times, would be anticipated (i.e., differences in elution times of an order magnitude or more). However, elution times for many of the experimental runs do not appear to correlate as expected with soil texture, with very similar reported elution times across most of the experiments for loam, silt loam, and clay soils (the sandy loam soil appeared to exhibit consistently longer elution times, presumably as a result of improper packing with the glass wool supporting material). Hence, the experimental data do not support the notion that the flow hydraulics are well controlled in these experiments.

- Introduction of PuriNOx and ordinary diesel fuel into the columns was accomplished by adding very small quantities of fuel $(100 \mu \mathrm{L})$ at the beginning of each experiment. Whether or not this method sufficiently emulates a realistic release scenario, or whether it is conservative or not, is unclear. A more defensible scenario is that of a body of NAPL floating on the water table. Under these conditions, complex constant-concentration boundary conditions would exist, affected by cosolvency mechanisms associated with multiple hydrocarbon constituents and surfactants. Although obviously more complex to design and interpret, such a column study 
would likely have provided a more convincing demonstration as to the expected behavior of the PuriNOx fuel mixture relative to that of ordinary diesel.

- The list of selected analytes for the experiments (PAH and diesel-range TPH) excluded BTEX compounds, which represent the most soluble and mobile components of diesel (Dunlap and Beckmann 1988) and are also likely to present the issue of greatest environmental concern from a water quality perspective. A comparison of travel time of BTEX species through a (larger) soil column between PuriNOx diesel and un-amended diesel would have helped to directly address the key issue as to whether or not the presence of surfactant species in the PuriNOx fuel could result in enhanced mobility of BTEX compounds.

- The soil columns used in the experiments were not characterized hydraulically in any way (this is perhaps related to the small column size). For a column-based contaminant mobility study, a tracer test using a conservative species is typically used to delineate a breakthrough concentration curve at the column outlet so that dispersion can be quantified. Contaminant breakthrough concentration curves are then normally developed and compared to the conservative tracer so that retardation/adsorption can be assessed. This approach was not followed in the series of soil column experiments undertaken on Lubrizol's behalf, so the data are less useful in terms of quantitatively validating the fugacity-based soil partitioning modeling.

Given the issues presented herein, it remains uncertain as to whether or not the U.S. EPA FIFRA guidelines for pesticide transport provide for a sufficiently robust experimental basis upon which a conceptual model of the environmental fate of PuriNOx fuel may be assessed. Moreover, given the small size of the experimental data set, the conclusion that there is "No statistically significant difference ... in the mobility of the indicator parameters between PuriNOx fuel and ULSD" (p. 102) appears to be phrased too strongly, especially in light of the problems listed above.

Furthermore, the available data for naphthalene behavior in ULSD versus the two PuriNOx fuels (Gen. 1 and 2) suggests that naphthalene indeed was transported to a greater degree in the PuriNOx fuel releases than in ULSD releases. For example, of 8 comparisons of PuriNOx fuels versus ULSD (i.e., 2 PuriNOx fuels and 4 soil types), naphthalene in column leachates (as percent of nominal dose) was greater from PuriNOx fuels than from ULSD in 7 cases (i.e., in $88 \%$ of the cases), and in 6 cases the naphthalene was 1.5- to 5-fold greater from PuriNOx fuels (p. 101). While this result may not be statistically significant according to parametric statistics (presumably as a result of high analytical variability and/or insufficient replication), it is certainly noteworthy and contrary to the conclusion stated by Lubrizol: "The above provides conclusive evidence that mobility and transport of PuriNOx fuel in soil will be similar to ULSD (p. 102)."

\section{III.2.5. Issues of Vadose Zone Transport Uncertainty}

As with other commercial fuel products, PuriNOx diesel fuel will, in many instances, likely be released into the subsurface environment via leaking underground and above-ground fuel tanks or through cumulative spillage. As such, a number of questions emerge about the behavior 
of the PuriNOx fuel mixture, as compared to that of ordinary diesel, in unsaturated soils in the event of such a release. Examples of questions that remain, in our judgment, still outstanding include the following:

- Given the presence of surfactant compounds in the additive package, will differences in surface tension between the PuriNOx fuel and ordinary diesel lead to significant differences in capillary forces that act on ganglia of NAPL within the vadose zone? At the interface between two fluids in a small capillary or pore space, the capillary pressure associated with the curved meniscus, $\mathrm{P}_{c}$, is defined by $\mathrm{P}_{\mathrm{c}}=2 \rho g \gamma \cos (\theta) / \mathrm{r}$, where $\rho$ is the fluid density, $\gamma$ the interfacial surface tension, $\theta$ the interfacial contact angle with the capillary wall, and $r$ the capillary (or pore) radius. This relationship plays a key role in determining when immiscible fluids such as water, NAPL, and air may displace one another in a porous media. Both $\gamma$ and, conceivably, $\theta$ are likely to be affected to some degree by the presence of surfactant agents, which can act to lower surface tension of a bulk fluid. As such, if these fluid properties are changed significantly in the PuriNOx fuel mixture as compared to that of ordinary ULSD fuel, it is possible that the PuriNOx fuel could exhibit different patterns of spatial distribution within the vadose zone (e.g., a greatly reduced surface tension might imply that the PuriNOx fuel could collect preferentially as a NAPL body on the water table, rather than as entrapped ganglia, as compared to ordinary diesel). Although interfacial surface tension values were quantified for select PuriNOx surfactant agents (Lubrizol internal memorandum dated July 25, 2003), a comparative analysis of bulk surface tension of PuriNOx fuel mixture to that of ordinary ULSD was not, to our knowledge, conducted, either experimentally or theoretically, so the issue of the potential impact of surface tension on the behavior of PuriNOx fuel in the vadose zone remains unresolved.

- Will surfactant compounds included in the additive package lead to the mobilization of residual hydrocarbon components entrapped in the vadose zone from prior releases? Potential releases of PuriNOx fuel or additive package components into the subsurface may introduce significant quantities of surfactant agents into soil pore water or, ultimately, groundwater, that could act to mobilize hydrocarbon compounds entrapped as NAPL ganglia or adsorbed to the soil matrix from past releases (e.g., a chronic leaking UST or cumulative spillage effects at a pumping station). Assessing the potential for this problem to occur is highly complex and site-specific, as the surfactants themselves may preferentially partition onto soils (competing with hydrocarbons for adsorption sites) or may partition into the NAPL phase. The potential importance of this issue remains unknown in the absence of experimental data from soil column experiments or field sites.

- What is the fate of hydrocarbon compounds that are dissolved within water droplets emulsified within the PuriNOx fuel mixture? Presumably, as a result of the large interfacial surface area afforded by the small size of the water droplets emulsified within PuriNOx fuel, hydrocarbon compounds found in diesel will reach equilibrium aqueous solubility concentrations within the droplets (neglecting the formation of micelles as a result of surfactants that may partition into the water phase as well). Taking into consideration the sizeable volumetric fraction of water in PuriNOx fuel, it 
is unclear as to whether or not the introduction of this impacted water into the subsurface will represent a significantly greater potential for adverse aquifer impact than would a release associated with ordinary diesel fuel. This issue is likely to be highly scenario-dependent.

\section{III.2.6. Capabilities for Routine Analytical Measurement of Environmental Distributions of PuriNOx Components}

Conceptual transport and fate models provide a framework for predicting contaminant distributions after releases, but actual measurements must be made to characterize contamination on a site-specific basis. Notably, most PuriNOx additive components are not compounds that are routinely analyzed by commercial analytical laboratories and in fact many are not amenable to standardized analytical methods developed by U.S. EPA and other agencies (in particular, methods relying on gas chromatography/mass spectrometry, or GC/MS). Therefore, in the event of a significant release, analysis for most components would require a custom analysis, typically requiring expensive analytical equipment (such as a liquid chromatograph/mass spectrometer, or LC/MS) that is rarely available in commercial environmental laboratories.

As indicated in the Lubrizol report, a few PuriNOx components can be analyzed by GC methods (including GC/MS), and some can be analyzed by ion chromatography or similar methods. However, many components and their hydrolysis products are too nonvolatile (due to polar functional groups and/or relatively high molecular weight) to be analyzed by GC and require analysis by LC/ESI(electrospray ionization)/MS (or possibly just ESI/MS).

It should be noted that many of the PuriNOx components have been in commercial use for 20 years or more (as discussed in the Lubrizol report), yet there are no regulatory monitoring requirements to assess their environmental release from these other uses.

\section{III.3. Data Uncertainty}

\section{III.3.1. Use of Structural Analogs to Characterize Key Environmental Toxicology and Biodegradation Parameters}

For a few PuriNOx components, structural analogs were used rather than the actual compounds to generate aquatic toxicity and aerobic biodegradation data. In general, the choice of analogs appears reasonable. However, some caution is warranted. Lubrizol's justification for this approach is essentially that the chemicals are structurally similar. However, the determination of similarity was subjective, in that no structure-activity data were provided to demonstrate, for example, that the analog acts biologically like the additive component it was chosen to represent.

For Component 1 (Gen. 1) and Component 1 (Gen. 2), a pair of analogs was chosen that had considerably shorter aliphatic side chains than the actual components (ca. $1000 \mathrm{amu}$ for the analogs vs. ca. 2000 amu for the actual components), which will render the analogs more soluble and possibly more biodegradable than the actual compounds. As an example of how aliphatic chain length can affect biodegradation, it has been found that anaerobic, alkane-degrading bacteria have very specific size ranges of alkanes that they can degrade (e.g., some species 
degrade only $\mathrm{C}_{6}$ to $\mathrm{C}_{8}$, whereas others degrade only $\mathrm{C}_{14}$ to $\mathrm{C}_{20}$; Spormann and Widdel 2000). Such differences in molecular weight also have the potential to affect uptake and toxicity.

Indeed, biodegradation data presented by Lubrizol suggest a marked effect on degradation of a seemingly minor structural difference. The two analogs for Component 1 (Gen. 1 and Gen. 2) differ from each other only in dimethyl versus diethyl substituents. Despite this relatively small difference, biodegradation of the dimethyl-substituted analog was $28 \%$ whereas biodegradation of the diethyl-substituted analog was only $7.6 \%$ (in 28 days). It seems unlikely that experimental variability would explain this difference in extent of biodegradation; indeed, interlaboratory variability in experimental conditions for tests of the two analogs would be expected to have favored the degradation of the analog that actually underwent less complete degradation (i.e., the diethyl-substituted analog). Specifically, the bacterial inoculum for the less-degraded analog was more than twice the concentration (in cells $/ \mathrm{mL}$ ) of that used for the other analog. Perhaps more importantly, the bacterial inoculum for the less-degraded analog was pre-adapted on the test compound for 14 days, whereas the other analog was not tested with a pre-adapted bacterial culture.

This apparent effect on biodegradation of a minor structural difference, and the welldocumented effects of minor structural differences on biodegradability for other compound classes [e.g., differences among xylene isomers; methylbenzene (i.e., toluene) versus ethylbenzene; Heider et al. 1998], merit consideration in light of Lubrizol's request that "future additive formulations utilizing similar chemical compositions all fall within the scope of this multimedia comparative analysis (p. 6)."

\section{III.3.2. Lack of Anaerobic Biodegradability Data for Many PuriNOx Components}

In most cases, assessments of biodegradation were based on the modified Sturm test (U. S. EPA 1987), which measures aerobic biodegradation of test substances by sewage sludge microorganisms. Although this assay may be an acceptable qualitative indicator of biodegradability under aerobic conditions, it has no relevance to anaerobic conditions, which are likely for some common release scenarios, namely, release into the subsurface via leaking USTs or release into low-energy sediment environments (such as marinas). Contaminated subsurface environments and quiescent sediments are often anaerobic. The metabolic pathways used by bacteria to degrade compounds aerobically are often completely different than anaerobic pathways; hydrocarbons provide an excellent example of this observation (e.g., Heider et al. 1998, Spormann and Widdel 2000). Whereas aerobic bacteria use oxygenases to attack a wide range of compounds, oxygenases are not functional in the absence of molecular oxygen (which serves as both a co-substrate and electron acceptor in mono- and dioxygenase reactions). Furthermore, anaerobic degradation rates are typically much slower than aerobic degradation rates; this has been widely reported for fuel hydrocarbons such as benzene (e.g., Alvarez et al. 2001) and alkanes. Thus, the concern is that the modified Sturm test may significantly overestimate biodegradability in the environment under relevant release scenarios. The unsubstantiated extrapolation of aerobic degradation results to anaerobic conditions occurs in the Lubrizol report, as on p. 106, when the results of an aerobic degradation study are summarized as follows: "In case of a spill, PuriNOx is expected to behave similar[ly] to diesel under both aerobic and anaerobic conditions." 
Anaerobic degradation data are available for Components 4 and 5 of Generation 1 and Components 3 and 4 of Generation 2, which have been more intensively studied because of their widespread commercial use.

Another noteworthy caveat about the modified Sturm test is that it should not be used to specify biodegradation rates in the environment even under aerobic conditions, as it involves bacterial populations and bacterial metabolic diversity (as a result of being exposed to municipal sewage) that are likely far greater than what would be expected in many natural environments, such as aquifers. The Lubrizol report appropriately did not attempt to extrapolate rates from results of the modified Sturm test.

\section{III.3.3. Lack of Knowledge About Biodegradation Pathways for Many PuriNOx Components: The Potential for Metabolites that Could Degrade Water Quality}

Ideally, biodegradation results in the complete mineralization of organic compounds to simple compounds such as $\mathrm{CO}_{2}$ and $\mathrm{H}_{2} \mathrm{O}$. The modified Sturm test addresses this by measuring the percent of $\mathrm{CO}_{2}$ produced from a compound rather than simply observing the disappearance of the compound from solution. For several of the PuriNOx components (or their analogs), the degree of mineralization to $\mathrm{CO}_{2}$ was only 0 to $28 \%$ in 28 days [e.g., Compounds 1, 2, 3, and 4 (or their analogs) in Generation 1 and Compound 1 in Generation 2]. This raises the question of whether problematic metabolites are accumulating during slow degradation, and whether a portion of a parent compound might be resistant to degradation (i.e., a degradable portion of the molecule could be mineralized to $\mathrm{CO}_{2}$ while the refractory portion would accumulate as a metabolic by-product).

Based on consideration of the structures of slowly degraded PuriNOx components, it is not obvious that anticipated degradation pathways would result in persistent metabolites that are more problematic than the parent compounds. However, it is very likely that intermediates of the degradation of high molecular weight compounds [such as Component 1 (Gen. 1 and Gen. 2) or Component 2 (Gen. 1)] would be more soluble than their parent compounds due to the shortening of aliphatic chains and the introduction of polar functional groups, such as carboxylic acids.

\section{III.3.4. Aquatic Toxicity Data Uncertainties}

Comprehensive aquatic toxicity testing of the PuriNOx additive package mixtures would have been challenging. However, given a choice, it would have been preferable to have aquatic toxicity data on the mixtures rather than on the individual components. If carefully conducted, the results from such tests would have yielded data of more immediate relevance than data on the individual compounds, as it is exposure to the mixture that will be sustained by organisms in the event of an accidental release.

One of the principal uncertainties regarding both the Generation 1 and Generation 2 PuriNOx additive packages is that there is no explicit evaluation of sediment toxicity that may occur from a release of either the 'neat' additive package, or of PuriNOx fuel, despite the clear and acknowledged tendency of many of the individual additive components to partition to and persist in soil and aquatic sediments. The potential effects of a release on sediment-dwelling organisms, on the species that rely on them (i.e., food-chain effects), or to sediment-driven bioaccumulation 
and the potential toxicity that may occur as a result, are not explicitly addressed. It appears to be inappropriate to apply an uncertainty factor of 10 to an aquatic toxicity measurement, and conclude that by so doing, one has adequately accounted for effects on benthic organisms (i.e., page 56). While aquatic species may depend on sediment-dwelling species, the organisms that inhabit these two environmental compartments are markedly different from one another. The concentrations that affect benthic organisms may have either no direct relationship, or no readilyquantified relationship, to the concentrations that affect aquatic species. As a result, no conclusions can be drawn from the information provided regarding the likely effects of a release of the PuriNOx materials on sediments and the biota that are dependent on them.

Significant uncertainties regarding the effects of the PuriNOx additive packages also arise from the nature of the aquatic toxicity data that were provided. Although in many cases the data are commendable in that they encompassed an evaluation of effects on multiple species, an acute exposure period with lethality as an endpoint was the only scenario considered. Given the demonstrated persistence of many of the PuriNOx components, this is clearly not the only exposure period of concern. Additionally, as amply demonstrated by data from many persistent organic chemicals that are present in the environment, non-lethal effects, which cannot be estimated from acute lethality data, can have profound toxicologic consequences to exposed organisms.

Acute aquatic toxicity criteria such as LC50 and/or EC50 values in the Lubrizol report are often well above the stated solubility of the compound - sometimes by orders of magnitude. Although the aquatic toxicity test methods and use of nominal concentrations appear reasonable, it is not valid to utilize nominal concentration data to derive LC50 and EC50 values without consideration of the physical limits on the solubility of the compound, and to do so calls into question the validity and utility of these criteria. Using such an approach tends to give a higher, incorrect, and often physically impossible value for the criterion. As a result, reliance on this approach leaves the determination of actual LC50 and/or EC50 values unresolved for all of the PuriNOx components with the exception of Component 3 (Gen. 2) and Component 4 (Gen. 2).

In information provided to the reviewers during a presentation by the Lubrizol Corporation, a statement was made to the effect that additive package components had not been evaluated experimentally for their potential to disrupt the endocrine system, and furthermore, by comparing the structure of known endocrine-disrupting compounds with the structure of the PuriNOx components, no endocrine-disrupting potential was expected. This argument is not correct, nor does it provide a sufficient basis for concluding that the PuriNOx components will not impact the endocrine system. For example, even if the discussion is limited to a single endocrine hormone receptor - the estrogen receptor (ER) - there is a very broad structural range of chemicals that elicit estrogenicity. The ER has the distinct ability to bind a number of structurally dissimilar chemicals (Brzozowski et al. 1997), apparently due in part to the fact that the ligand-binding pocket of the ER has a much greater volume than the endogenous ligand (17 $\beta$-estradiol). In practical terms, this means that structural comparisons do not provide a reasonable estimation of the ability of a chemical to interact with the ER, the most commonly studied target of endocrineactive substances.

In a number of places throughout the Multimedia evaluation document, Lubrizol equates limited water solubility with minimal or no toxicity. This relationship is not necessarily correct. 
There are a number of well-known examples in which minimally soluble environmental contaminants have significant toxicity, and to reach a conclusion regarding the relationship between solubility and toxicity in the absence of data is not justified. Consequently, it is not appropriate or informative to invoke limited solubility as a reason for not providing toxicity data, or to support assumptions that it is appropriate to provide aquatic toxicity data from a more soluble analog because of its presumed greater toxicity. This caveat is relevant to the following statement taken from the Lubrizol report: "Read across data is applied only if there is a high level of confidence that the data obtained represents the most conservative estimate of toxicity... It is appropriate to use data available for a lower molecular weight and more water soluble component as read across for a higher molecular weight and less water soluble chemical. It is well documented in the scientific literature that less water soluble and higher molecular weight chemicals have low bioavailability and are likely to be less toxic" (p. 30).

For example, some of the Generation 1 additive package components are manufactured in base oil to decrease viscosity of the mixture. The amount of base oil that remains in the additive package may range from 25-30\%. The base oils are described as "paraffinic or naphthenic.... Both the paraffinic and naphthenic hydrocarbons in the $\mathrm{C}_{15}$ to $\mathrm{C}_{50}$ range are too water insoluble to cause aquatic toxicity." Although summary data cited and provided by Lubrizol (Concawe 1997) indicate that a range of base oil formulations are not particularly toxic when evaluated for lethality or reproductive effects, low-solubility compounds can have both direct irritant effects on gill tissue, and/or may cause non-specific toxicity, e.g., by interfering with oxygen uptake or by physically disrupting membranes - toxicological endpoints not explicitly evaluated in the Concawe (1997) data.

When the additive package components are blended with fuel, the mixture may act similarly to chemically-dispersed oil if released to an aquatic environment. In its evaluation of oil spill dispersants, the National Academy of Sciences (NAS 1989) noted that, for those dispersants studied to date, laboratory data demonstrate that in general, the acute toxicity of dispersed and untreated oil are similar. This indicates that for these surface-active agents, there do not appear to be additive or synergistic effects on aquatic organisms upon exposure to the fuel-dispersant mixture. Extrapolating this conclusion to a spill of PuriNOx fuel may well be appropriate, although we do not have specific data to support such a conclusion at this time. However, the NAS (1989) report also pointed out that chemically-dispersed oil slicks can affect different organisms than oil (fuel) alone. Surfactants and dispersants - such as Components 1 and 2 (Gen. 1) - released in conjunction with fuel hydrocarbons to aquatic environments, have the potential to alter the distribution of spilled fuel, and thus alter the group of organisms that may be adversely affected. Fuel-surfactant mixtures can be expected to partition deeper into the water column than fuel released alone, causing relatively greater exposure to organisms in subsurface waters. This suggests that the actual impacts on aquatic species from a spill may well depend on the timing of the spill relative to the reproductive cycle of aquatic species, as eggs and larvae inhabit different regions of the water column at different times of their life cycle. Additionally, the NAS (1989) noted that if a surfactant-fuel spill occurs in shallow waters with poor water circulation, sediment-dwelling organisms may be affected sooner than from a spill of nondispersed oil. 


\section{III.4. Cleanup Technologies}

At some point, releases of PuriNOx fuel to both surface waters and the subsurface environment must be anticipated; however, the cleanup discussion provided by Lubrizol did not address subsurface releases explicitly. We believe that cleanup responses to releases of the (fully mixed) fuel product to the subsurface will likely entail the application of the same remediation approaches that would normally be applied to a ULSD spill. These would include, for example, tank removal and source area excavation, free product skimming, soil vapor extraction, and, where applicable, monitored natural attenuation. Whether the more water soluble components included in PuriNOx additive package would form significant groundwater plumes, either as a result of partitioning from free product spills or as a result of a releases of tank bottom water enriched in soluble components, and would require pump-and-treat remediation, is unclear and highly scenario dependent. Whether or not such plumes could be easily identified is also uncertain. Presumably, the low concentrations of soluble additive components in the PuriNOx fuel mixture would mitigate this concern to some extent.

The release of PuriNOx fuel to surface waters, particularly marine waters, may present a more difficult problem. To the extent that surface water releases and cleanup are discussed in the Lubrizol report and Supplemental Information, Lubrizol indicates that the PuriNOx mixture of diesel and additives will exist as a separate phase film at the water surface, where it is believed that the emulsion will break up over time into diesel and water, where it can be removed in the same manner as ordinary diesel fuel released into surface water.

The PuriNOx additive package, which contains emulsifiers and dispersants, depends on both lipophilic and hydrophilic components to keep water suspended in fuel. Water-in-oil (lipophilic) emulsions are very stable and may persist for months, or even years, after a spill. In oil spills that have occurred in marine surface waters, water-in-oil emulsions consisting of 50 to 80 percent water form a grease-like, reddish-brown material frequently referred to as "mousse." Additionally, surfactants reduce the water surface tension, which can result in increased hydrocarbon movement into the water column. If mousse formation should occur, or if diesel fuel and additive components move downward into the water column, cleanup may be more problematic. Cleanup options that include mechanical containment and collection may not be practicable in either of these situations. Further, aquatic organisms may suffer adverse impacts from dispersant compounds, and from surface active PuriNOx components that may behave similarly to dispersants (Section III.3.4). The various types and limitations of oil spill cleanup methods have been detailed by the National Academy of Sciences (1989). Notably, Lubrizol has stated in a Supplemental Information memorandum (September 5, 2003) that, based on their experience with PuriNOx additive packages, mousse formation is highly unlikely in any release scenario involving additive concentrates; however, it is still uncertain how a release of PuriNOx fuel would react under the mixing and shear conditions that occur during wave action in surface waters. 


\section{III.5. Potential for Environmental and Water Resource Impacts Relative to ULSD}

\section{III.5.1. Taste and Odor Concerns}

As reported by Lubrizol, zero aerobic or anaerobic degradation was observed in 28 days for Component 4 (Gen. 1), which is also included in Generation 2. Although this compound does not appear to be highly toxic, it does have a distinctive odor and appreciable water solubility (between 12 and $18 \mathrm{mg} / \mathrm{L}$ ). Therefore, it is possible that this compound may persist in water after release in the environment (particularly in the subsurface, since it may tend to volatilize from surface water). Notably, Lubrizol indicates that this compound will hydrolyze in water (half-life of 7 days at $\mathrm{pH} 7$ ). If so, this would mitigate concerns about persistence. However, it is difficult to reconcile the lack of aerobic biodegradation (in 28 days) with relatively rapid hydrolysis at neutral $\mathrm{pH}$, as the expected organic hydrolysis product should be relatively easy to degrade under aerobic conditions.

This biologically-recalcitrant component is commonly used as a cetane improver in diesel fuels, although the concentration added to PuriNOx fuel is more than an order of magnitude higher than that added to ULSD. With regard to Component 4 (Gen. 1), Lubrizol has provided the following information: "A test plan is being prepared by the American Chemistry Council (ACC) Lubricant Additives Industry Consortium under the U.S. EPA High Production Volume (HPV) Chemical Challenge program. This will enable a complete environmental dossier to be developed based on physical-chemical and toxicity testing on this chemical which will be publicly available on the EPA HPV web site."

There are also potential taste/odor concerns with the two substituted alkanolamine components or hydrolysis products associated with Components 1 and 3 (Gen. 1) and Components 1 and 2 (Gen. 2). These compounds have relatively high water solubility and a distinctive, fishy odor. Partitioning experiments with PuriNOx fuels (p. 105) indicated that one of these compounds could have an appreciable aqueous concentration (23\% of the added mass). Although both compounds are apparently degradable under aerobic conditions (information for one compound is available only in its Material Safety Data Sheet, which is of unknown reliability), there is no information on their anaerobic biodegradability.

\section{III.5.2. Concerns with Eutrophication Resulting from Releases of Component 5 (Generation 1)}

One concern with environmental releases of PuriNOx additive package or PuriNOx fuels is that the two constituents of Component 5 (Gen. 1), which is also included in Gen. 2, will promote eutrophication in surface waters. These constituents are highly soluble in water and are known to serve as fertilizers that can promote algal blooms. Whether eutrophication will result from PuriNOx releases will depend in large part on the potential for dilution in the affected surface water body (e.g., flow rate of a river, or advection rates and volumes of estuaries and lakes). Also, this issue will clearly be of more concern for releases of the additive package than for PuriNOx fuel.

Since the anionic constituent of Component 5 is regulated in groundwater, subsurface releases could also be a concern. However, if the subsurface release is of PuriNOx fuel, it is 
likely that this compound will serve as an electron acceptor for anaerobic hydrocarbon degradation and will not persist.

\section{III.5.3. Possible Enhanced Solubility and Mobility of Diesel Hydrocarbons}

Considering the surfactant properties of many PuriNOx components, an obvious concern about releases of PuriNOx fuels is that aqueous concentrations of diesel hydrocarbons (e.g., naphthalene and BTEX) could be enhanced relative to the concentrations resulting from releases of ULSD. As discussed previously (Sections III.2.1, III.2.4, and III.2.5), PuriNOx components could facilitate mobilization of residual hydrocarbons entrapped in the vadose zone and could also enhance the solubilization of hydrocarbons in the saturated zone. Existing fugacity models cannot address these issues, and the empirical partitioning and soil column experiments reported by Lubrizol do not resolve them, because of limitations in experimental design and/or equivocal results (Sections III.2.1, III.2.2, III.2.4, and III.2.5). Thus, questions about enhanced solubility of diesel hydrocarbons after releases of PuriNOx fuel remain unresolved. 


\section{Summary and Conclusions}

\section{IV.1. Conformance of Lubrizol's Data Package to SWRCB Evaluation Criteria}

Lubrizol has taken the precedent-setting challenge of being the first company to bring a new fuel to market in California under the Senate Bill 989 requirements for multimedia assessment. In this capacity, Lubrizol has made a significant good-faith effort to provide appropriate data to meet these requirements. This is a very complex challenge that has been undertaken with minimal guidance from those agencies with regulatory oversight responsibilities. The company's staff has been very cooperative, responsive, and open with the data that are available to them, including proprietary information. The PuriNOx Fuel Multimedia Evaluation Final Report and Supplemental Information addressed all the major elements in the SWRCB data package guidance.

A further burden is the lack of general environmental information on many of the components in the PuriNOx additive package, in spite of the widespread industry use of most of these components. Lubrizol has provided considerable physical-chemical and toxicological data on additive components and/or structurally-related analogs, however, all of the toxicological and environmental behavior information needed to conduct a thorough evaluation is not available. It would be prohibitive to require a single small company to fill these data gaps in order to bring a potentially beneficial fuel to market in California. Nevertheless, these constraints do not diminish any concerns that have been raised during this review; many of the criticisms and comments herein are aimed at improving the data submittals for future fuel multimedia assessments and fully informing decision makers regarding knowledge gaps.

\section{IV.2. Considerations About the Fugacity Modeling Approach}

The Lubrizol multimedia evaluation includes a steady-state, fugacity-based simulation of the distribution of PuriNOx additive components in a landscape consisting of air, water, soil, and sediment compartments. The primary value of this multimedia simulation approach is that it provides useful diagnostic information on the likely distribution of a compound in different media as a result of alternative release scenarios, environmental properties, and various transport and fate processes.

There are limitations to the compartmental modeling approach, especially when dealing with a broad range of potential release conditions and fuel mixtures with complex chemistry. In particular, fugacity-based multimedia modeling is only applicable to dilute concentrations of a given compound in a completely mixed, homogeneous environmental compartment. Moreover, such modeling does not deal with chemical interactions between a contaminant and host media. At present, there are no methods available to make quantitative predictions of the relevant environmental properties of all surfactant classes, and of the complex interactions between surfactants and hydrocarbon fuel components in a multimedia environment. 


\section{IV.3. Discrepancies Between Empirical Partitioning Data and Fugacity Predictions}

Although fugacity-based models may provide a semi-quantitative idea of the partitioning of individual PuriNOx components in the environment, empirical studies provide more reliable (although not necessarily quantitative) indicators of complex partitioning behavior. The Lubrizol report presents data for the partitioning of PuriNOx and PuriNOx fuel components between water and a complex NAPL. Overall, the empirical results suggest that fugacity models may be less than reliable even in systems that are far less complex than the natural environment. For example, in a study in which PuriNOx additive packages (Generations 1 and 2) were added to water and allowed to equilibrate, Component 3 (Gen. 1) was present in water at over 700,000 times its estimated water solubility. Although the explanation may be that this compound was "dispersed," not truly dissolved, in the aqueous phase, this distinction is of dubious importance from the applied environmental perspective - both dissolved and dispersed fractions of a compound will be transported in the aqueous phase and can affect organisms in contact with that phase. The fugacity modeling presented by Lubrizol did not account for dispersion of compounds into the aqueous phase, only dissolution.

Empirical results were also difficult to explain in partitioning experiments designed to answer the question: will diesel hydrocarbons, such as BTEX, have enhanced water solubility after releases of PuriNOx fuel relative to ULSD? In these experiments, TPH and BTEX had opposite behaviors. TPH concentrations in water were 7 to 22 times higher for PuriNOx fuels than for ULSD; however, BTEX concentrations were typically 2 to 3 times lower for PuriNOx fuels than for ULSD. It seems highly implausible that surfactants would enhance the solubility of one class of hydrocarbons while reducing the solubility of another class. Lubrizol suggests that the confounding behavior could be an analytical artifact, since TPH and BTEX were measured by different methods that may have been variously affected by dispersed emulsion droplets. Regardless, it is questionable whether these data are sufficiently reliable to include in a conceptual transport and fate model.

\section{IV.4. Considerations About the Comparison of PuriNOx Components to MTBE}

The Lubrizol report stressed the point that "the PuriNOx additive package components will not adversely impact the environment like MTBE". The predominant concept underlying the favorable comparison of PuriNOx components to MTBE is that many PuriNOx components have low water solubilities and will thus be effectively immobile in the subsurface environment. While this is a valid point, several points are noteworthy with respect to water solubility and PuriNOx components: (a) for releases to surface water, low solubility cannot necessarily be equated with low environmental concern, as insoluble, sediment-associated compounds may be ingested by benthic organisms and bioaccumulate, (b) there are PuriNOx components or hydrolysis products that are somewhat soluble that may persist in the subsurface, and (c) in addition to considering PuriNOx components themselves, we must also consider the potential effects of PuriNOx components on enhancing the solubility of toxic ULSD compounds (such as BTEX hydrocarbons). 


\section{IV.5. Representativeness of Soil Column Experiments}

The mobility study of hydrocarbon components in soil columns undertaken by Lubrizol, in our opinion, represented the best opportunity to compare the behavior of PuriNOx fuel against that of ordinary ULSD within a multimedia context short of an actual field experiment. The soil column studies were conducted by an accredited independent laboratory on behalf of Lubrizol in accordance with U.S. EPA FIFRA and GLP guidelines, following protocols designed to study the mobility and behavior of pesticides in the environment. However, the experiments were not representative of likely release scenarios and critical data were not measured. Specific problems with the soil column experiments include the following: (a) the soil columns $(10-\mathrm{mL}$ pipettes filled with test soils to a depth of $3 \mathrm{~cm}$ ) were far too small to exclude possible edge effects exerted by the walls of the pipettes on solute transport, (b) the application of very small quantities of fuel $(100 \mu \mathrm{L})$ to the columns arguably did not emulate likely release scenarios, (c) the list of selected analytes excluded BTEX compounds, which are likely to present the issue of greatest environmental concern from a water quality perspective, and (d) the soil columns were not characterized hydraulically. Furthermore, the available data for naphthalene behavior in ULSD versus the two PuriNOx fuels (Gen. 1 and 2) suggests that naphthalene indeed was transported to a greater degree in the PuriNOx fuel releases than in ULSD releases (Section III.2.4). While this result may not have been statistically significant, it is certainly noteworthy and contrary to the conclusion stated by Lubrizol: "The above provides conclusive evidence that mobility and transport of PuriNOx fuel in soil will be similar to ULSD."

\section{IV.6. Vadose Zone Transport Uncertainty}

As with other commercial fuel products, PuriNOx diesel fuel will, in many instances, likely be released into the subsurface environment via leaking underground and above-ground fuel tanks or through cumulative spillage. As such, a number of questions emerge about the behavior of the PuriNOx fuel mixture, as compared to that of ordinary diesel, in unsaturated soils in the event of such a release. Examples of questions that remain, in our judgment, still outstanding include the following: (a) will surfactant compounds included in the additive package lead to the mobilization of residual hydrocarbon components entrapped in the vadose zone from prior releases, and (b) what is the fate of hydrocarbon compounds that are dissolved within water droplets emulsified within the PuriNOx fuel mixture?

\section{IV.7. Capabilities for Routine Analytical Measurement of PuriNOx Components}

Conceptual transport and fate models provide a framework for predicting contaminant distributions after releases, but actual measurements must be made to characterize contamination on a site-specific basis. Notably, most PuriNOx additive components are not compounds that are routinely analyzed by commercial analytical laboratories and in fact many are not amenable to standardized analytical methods developed by U.S. EPA and other agencies. Specifically, many components and their hydrolysis products are too nonvolatile (due to polar functional groups and/or relatively high molecular weight) to be analyzed by gas chromatography techniques. Therefore, in the event of a significant release, analysis for most components would require a custom analysis, typically requiring expensive analytical equipment (such as LC/MS) that is 
rarely available in commercial environmental laboratories. It should be noted that many of the PuriNOx components have been in commercial use for 20 years or more, yet there are no regulatory monitoring requirements to assess their environmental release from these other uses.

\section{IV.8. Use of Structural Analogs to Characterize Aquatic Toxicity and Biodegradation}

For a few PuriNOx components, structural analogs were used rather than the actual compounds to generate aquatic toxicity and aerobic biodegradation data. In general, the choice of analogs appears reasonable. However, some caution is warranted. In fact, certain biodegradation data presented by Lubrizol suggest a marked effect on degradation of a seemingly minor structural difference.

\section{IV.9. Lack of Anaerobic Biodegradability Data for Many PuriNOx Components}

In most cases, assessments of biodegradation were based on the modified Sturm test, which measures aerobic biodegradation of test substances by sewage sludge microorganisms. Although this assay may be an acceptable qualitative indicator of biodegradability under aerobic conditions, it has no relevance to anaerobic conditions, which are likely for some common release scenarios, namely, release into the subsurface via leaking USTs or release into lowenergy sediment environments (such as marinas). Furthermore, anaerobic degradation rates are typically much slower than aerobic degradation rates. Thus, the concern is that the method used to generate most of the biodegradation data presented by Lubrizol may significantly overestimate biodegradability in the environment under relevant release scenarios.

\section{IV.10. Potential for Metabolites that Could Degrade Water Quality}

Slow biodegradation observed for some compounds raises the question of whether problematic metabolites are accumulating during slow degradation, and whether a portion of a parent compound might be resistant to degradation, leading to the formation of refractory metabolic by-products. Based on our consideration of the structures of slowly degraded PuriNOx components, it is not obvious that anticipated degradation pathways would result in persistent metabolites that are more problematic than the parent compounds.

\section{IV.11. Aquatic Toxicity Data Uncertainties}

Comprehensive aquatic toxicity testing of the PuriNOx additive package mixtures would have been challenging. Given a choice, it would have been preferable to have aquatic toxicity data on the mixtures rather than on the individual components. However, if carefully conducted, the results from such tests would have yielded data of more immediate relevance than data on the individual compounds, as it is exposure to the mixture that will be sustained by organisms in the event of an accidental release. Among the concerns about the toxicity data are the following: (a) there is no explicit evaluation of sediment toxicity that may occur from a release of either the 'neat' additive package, or of PuriNOx fuel, despite the clear and acknowledged tendency of many of the individual additive components to partition to and persist in soil and aquatic 
sediments, (b) an acute exposure period with lethality as an endpoint was the only scenario considered, (c) acute aquatic toxicity criteria such as LC50 and/or EC50 values in the Lubrizol report are often well above the stated solubility of the compound, (d) from data presented, there is no basis for concluding that the PuriNOx components will not impact the endocrine system, and (e) Lubrizol equates limited water solubility to minimal or no toxicity; this relationship is not necessarily correct, and it was inappropriate to invoke limited solubility as a reason for not providing toxicity data, or to support assumptions that it is reasonable to provide aquatic toxicity data from a more soluble analog because of its presumed greater toxicity.

\section{IV.12. Cleanup of Releases}

At some point, releases of PuriNOx fuel to both surface waters and the subsurface environment must be anticipated. We believe that cleanup responses to releases of the (fully mixed) fuel product to the subsurface will likely entail the application of the same remediation approaches that would normally be applied to a ULSD spill. The release of PuriNOx fuel to surface waters, particularly marine waters, may present a more difficult problem. In oil spills that have occurred in marine surface waters, water-in-oil emulsions consisting of 50 to 80 percent water form a grease-like, reddish-brown material frequently referred to as "mousse." Additionally, oil-in-water surfactants reduce the water surface tension, which can result in increased hydrocarbon movement into the water column. If mousse formation should occur, or if diesel fuel and additive components move downward into the water column, cleanup may be more problematic. Cleanup options that include mechanical containment and collection may not be practicable in either of these situations.

\section{IV.13. Principal Considerations About Potential Water Resource Impacts}

Based on a projected market share of 40 million gallons of PuriNOx per year, the volume of this additive package moving into the state will be on the order of 0.8 to 1.2 million gallons per year (by the year 2012). This is a relatively minor amount compared to the volume of chemicals with more severe environmental consequences that are routinely transported in this manner. Although there is a potential for a release of the bulk additive package components during transport into California, the likelihood of a bulk additive package component release during transport is relatively small.

Release of PuriNOx fuel from leaking USTs poses the most likely release scenario; thus, an understanding of the differences in behavior between a ULSD subsurface release and a PuriNOx fuel subsurface release will be key to effective management of this new fuel. Since one of the initial deployments of PuriNOx fuel will be to port operations, there may be a release scenario in which blended fuel is released to the surface of marine waters, either through surface runoff or through a bulk spill. The impact of the additive package on the behavior of diesel fuel in a marine environment, as well as potential interactions between saltwater and additive package components, are important considerations.

\section{IV.13.1. Taste and Odor Concerns}

As reported by Lubrizol, zero aerobic or anaerobic degradation was observed in 28 days for Component 4 (Gen. 1), which is also included in Generation 2. Although this compound does 
not appear to be highly toxic, it does have a distinctive odor and appreciable water solubility (between 12 and $18 \mathrm{mg} / \mathrm{L}$ ). Therefore, it is possible that this compound may persist in water after release in the environment (particularly in the subsurface, since it may tend to volatilize from surface water). Notably, Lubrizol indicates that this compound will hydrolyze in water (half-life of 7 days at $\mathrm{pH} 7$ ). If so, this would mitigate concerns about persistence. However, it is difficult to reconcile the lack of aerobic biodegradation with relatively rapid hydrolysis at neutral $\mathrm{pH}$, as the expected organic hydrolysis product should be relatively easy to degrade under aerobic conditions.

This biologically-recalcitrant component is commonly used as a cetane improver in diesel fuels, although the concentration added to PuriNOx fuel is more than an order of magnitude higher than that added to ULSD. With regard to Component 4 (Gen. 1), Lubrizol has provided the following information: "A test plan is being prepared by the American Chemistry Council (ACC) Lubricant Additives Industry Consortium under the U.S. EPA High Production Volume (HPV) Chemical Challenge program. This will enable a complete environmental dossier to be developed based on physical-chemical and toxicity testing on this chemical which will be publicly available on the EPA HPV web site."

There are also potential taste/odor concerns with the two substituted alkanolamine components or hydrolysis products associated with Components 1 and 3 (Gen. 1) and Components 1 and 2 (Gen. 2). These compounds have relatively high water solubility and a distinctive, fishy odor. Although both compounds are apparently degradable under aerobic conditions, there is no information on their anaerobic biodegradability.

\section{IV.13.2. Concerns with Eutrophication Resulting from Releases of Component 5 (Generation 1)}

One concern with environmental releases of PuriNOx additive package or PuriNOx fuels is that the two constituents of Component 5 (Gen. 1), which is also included in Gen. 2, will promote eutrophication in surface waters. These constituents are highly soluble in water and are known to serve as fertilizers that can promote algal blooms. Whether eutrophication will result from PuriNOx releases will depend in large part on the potential for dilution in the affected surface water body.

Since the anionic constituent of Component 5 is regulated in groundwater, subsurface releases could also be a concern. However, if the subsurface release is of PuriNOx fuel, it is likely that this compound will serve as an electron acceptor for anaerobic hydrocarbon degradation and will not persist.

\section{IV.13.3. Possible Enhanced Solubility and Mobility of Diesel Hydrocarbons}

Considering the surfactant properties of many PuriNOx components, an obvious concern about releases of PuriNOx fuels is that aqueous concentrations of diesel hydrocarbons (naphthalene and BTEX, in particular) could be enhanced relative to the concentrations resulting from releases of ULSD. As discussed previously (Sections III.2.1, III.2.4, and III.2.5), PuriNOx components could facilitate mobilization of residual hydrocarbons entrapped in the vadose zone and could also enhance the solubilization of hydrocarbons in the saturated zone. Existing fugacity models cannot address these issues, and the empirical partitioning and soil column 
experiments reported by Lubrizol do not resolve them, because of limitations in experimental design and/or equivocal results (Sections III.2.1, III.2.2, III.2.4, and III.2.5). Thus, questions about enhanced solubility of diesel hydrocarbons after releases of PuriNOx fuel remain unresolved. 


\section{References}

Alvarez, P. J. J., Ruiz-Aguilar, G. M. L., Fernandez-Sanchez, J. M., Kim, D., Beller, H. R., and Kane, S. R. 2001. Chapter 3: Effect of ethanol and MTBE on BTEX biodegradation in the saturated zone: Kinetic studies. In Environmental Assessment of the use of ethanol as a fuel oxygenate. Lawrence Livermore National Laboratory, Livermore, Calif. (UCRLAR-145380).

Boethling, R. S. and Mackay, D. 2000. In Handbook of Property Estimation Methods for Chemicals, Chapter 17, Lewis Publishers, Boca Raton.

Brzozowski, A. M., Pike, A. C., Dauter, Z., Hubbard, R. E., Bonn, T., Engstrom, O., Ohman, L., Greene, G. L., Gustafsson, J. A., and Carlquist, M. 1997. Molecular basis of agonism and antagonism in the oestrogen receptor. Nature 389:753-758.

ChevronTexaco. 2003. Proformix Fuel Application Requirements: http://www.chevron.com/prodserv/ fuels/proformix/customer_information.shtml

Concawe. 1997. Lubricating Oil Basestocks, Product Dossier 97/108, prepared by Concawe's Petroleum Products and Health Management Groups.

Dunlap, L. E., and Beckmann, D. D. 1988. Soluble hydrocarbon analysis from kerosene/diesel type hydrocarbons. In Proceedings of Petroleum Hydrocarbons and Organic Chemicals in Groundwater, vol. 1, pp. 37-45. National Water Well Association, Dublin, OH.

Heider, J., Spormann, A. M., Beller, H. R., and Widdel, F. 1998. Anaerobic bacterial metabolism of hydrocarbons. FEMS Microbiol. Reviews 22:459-473.

National Academy of Sciences. 1989. Using Oil Spill Dispersants on the Sea. National Research Council, National Academy of Sciences. National Academies Press, Washington, DC.

Spormann, A. M., and Widdel, F. 2000. Metabolism of alkylbenzenes, alkanes, and other hydrocarbons in anaerobic bacteria. Biodegradation 11:85-105.

U. S. Environmental Protection Agency. 1987. Ready biodegradability: Modified Sturm Test. 40 CFR 796.3260 . 


\section{Author Biographies}




\section{Harry R. Beller, Ph.D.}

Environmental Microbiology/Analytical Chemistry

Dr. Harry Beller is an environmental scientist at Lawrence Livermore National Laboratory specializing in environmental microbiology and chemistry. Dr. Beller has performed extensive research on the anaerobic biodegradation of organic contaminants (including BTEX hydrocarbons and explosives); this research has included physiological, biochemical, and molecular studies, as well as the isolation and characterization of biodegrading bacteria. He has also developed mass spectrometric techniques, including liquid chromatography/tandem mass spectrometry methods, for ultra-sensitive and specific detection of signature microbial metabolites and selected contaminants. He has over 20 peer-reviewed journal publications on environmental microbiology and chemistry since 1992. He is a coauthor of the report "Use of Ethanol as a Fuel Oxygenate: Subsurface Fate and Transport of Gasoline Containing Ethanol."

He earned a B.A. in Environmental Science from Wesleyan University (CT), an M.S. in Chemical Oceanography from Oregon State University, and a Ph.D. in Civil (Environmental) Engineering from Stanford University. Dr. Beller is also an Adjunct Associate Professor in the Department of Chemical Engineering and Applied Chemistry at the University of Toronto (Canada). Awards given to Dr. Beller include the American Chemical Society award for Graduate Students in Environmental Chemistry, and a Switzer Foundation Environmental Fellowship.

\section{Brendan P. Dooher, Ph.D.}

Systems Analysis/Risk Assessment

Dr. Dooher is a systems analyst and risk assessor with Lawrence Livermore National Laboratory. He has studied probabilistic risk assessment and environmental systems under the direction of Professor Bill Kastenberg, Daniel M. Tellep Distinguished Professor of Engineering. Dr. Dooher has worked extensively with California public agencies to assess the threat to public water supplies and resources from leaking underground fuel tank sites and MTBE. He has helped create the interactive GIS site, GeoTracker, to help regulators, responsible parties, and the public assess water supplies vulnerability more cost effectively and develop a "living groundwater model" for California. He is a coauthor of the report "Health and Environmental Assessment of the Use of Ethanol as a Fuel Oxygenate" presented to the California Environmental Policy Council in response to executive order D-5-99.

He received his Ph.D. in Mechanical Engineering in 1998 from the University of California, Los Angeles.

\section{Linda C. Hall, Ph.D.}

Aquatic Toxicology

Dr. Linda Hall is an environmental scientist at Lawrence Livermore National Laboratory, with expertise in aquatic toxicology, especially reproductive endocrine disruption of aquatic contaminants in fish. She has identified a novel class of herbicide as estrogen agonists. She had performed research regarding the reproductive toxicity of fish and the biochemical mechanisms of reproductive toxicity e.g., tissue-specific alterations in expression of p450aromatase and estrogen receptor mRNA detected by quantitative PCR. Dr. Hall also has expertise in human health risk assessment, routes and mechanisms of human exposure to environmental 
contaminants and cancer risk, and non-cancer health effects from exposure to chemical and biological contaminants.

Received a B.A. in Ecosystems Analysis, Antioch College, Yellow Springs, OH. in 1977, and M.A. in Toxicology, San Jose State University, San Jose, CA. in 1987, and Ph.D. Ecology (Ecological Toxicology), University of California, Davis, CA. In 2002.

\section{David W. Layton, Ph.D.}

\section{Environmental Risk Assessment}

David Layton is a senior scientist in the Environmental Science Division at Lawrence Livermore National Laboratory. During the early part of his 27-year career at LLNL, his research focused on the health and environmental impacts of geothermal energy development in the western U.S. Later, he led a major assessment of the information available on conventional ordnance (i.e., explosives and propellants) in order to support studies of the impacts of weapons demilitarization. He was also one of the lead investigators of a DoD-funded project to revise the field-water quality standards used by the U.S. military. After these projects, his research emphasis gradually shifted to the development of quantitative risk assessments of environmental contaminants. He has prepared risk assessments of hazardous gas releases, residual ground-water contamination at a Superfund site, plutonium-contaminated soils, heterocyclic amines in cooked foods, and nuclear wastes dumped in the Arctic Ocean. In addition, he has worked to improve exposure parameters for use in risk assessments. For example, he developed an innovative, metabolically-based method for determining breathing rates and conducted research on the re-suspension of particles indoors. More recently, he has initiated projects dealing with environmental assessments of transportation fuels and associated additives such as ethanol and MTBE. He is a coauthor of the report "Health and Environmental Assessment of the Use of Ethanol as a Fuel Oxygenate" presented to the California Environmental Policy Council in response to executive order D-5-99.

He has B.A. degree in Earth Science and a Ph.D. in Water Resources Administration.

\section{Alfredo A. Marchetti, Ph.D.}

\section{Environmental Chemistry}

Dr. Alfredo Marchetti is an environmental chemist at Lawrence Livermore National Laboratory with experimental expertise in analytical chemistry, radiochemistry, and nuclear chemistry, including applications to environmental problems. He has developed extraction and analytical methods for a variety of environmental samples and has experience in accelerator mass spectrometry. He has designed and implemented Monte Carlo calculations using transport codes. He has implemented and applied chemical property estimation methods and screening models to assess the fate of fuel-related compounds in the environment and conducted biodegradation experiments for potential diesel fuel additives. He is a coauthor of the report "Health and Environmental Assessment of the Use of Ethanol as a Fuel Oxygenate" presented to the California Environmental Policy Council in response to executive order D-5-99.

Received a License degree in analytical chemistry from the University of Buenos Aires, Argentina in 1984, and a Ph.D. in nuclear chemistry from the University of Maryland at College Park in 1991. 


\section{Walt W. McNab, Jr., Ph.D., R.G.}

Hydrology/Geochemistry

Walt McNab is an environmental scientist with the Environmental Restoration Division at Lawrence Livermore National Laboratory. His research interests include groundwater flow and transport modeling, aqueous geochemistry and geochemical modeling, simulation of multispecies reactive transport, and statistical studies of groundwater contamination. He has conducted research on specialized groundwater treatment technologies, including electroosmosis and in-well catalytic reductive dehalogenation, in both field and laboratory settings, leading to two U.S. patents. He has authored papers on a variety of groundwater-related topics in scientific journals such as Environmental Science \& Technology, Ground Water, Ground Water Monitoring \& Remediation, and Water Resources Research. He is a coauthor of the report "Health and Environmental Assessment of the Use of Ethanol as a Fuel Oxygenate" presented to the California Environmental Policy Council in response to executive order D-5-99.

Dr. McNab is a registered geologist and holds an A.B. in geology and an M.S. and Ph.D. in hydrogeology from U.C. Berkeley. In addition to his role as a scientist at Lawrence Livermore Lab, he has also worked as an environmental consultant with Kennedy/Jenks Consultants, Exponent - Failure Analysis Associates, and CH2MHill.

\section{David W. Rice}

\section{Comprehensive Environmental Assessment/Fuels Fate and Transport}

Mr. Rice is the Environmental Chemistry and Biology Group Leader for the Lawrence Livermore National Laboratory (LLNL) Environmental Protection Department. He is an expert in the fate and transport of contaminants in subsurface sediments, ground water, and the marine environment. During his 28 years at LLNL, Mr. Rice has participated in the management of the LLNL superfund sites and directed and performed research on environmental decision-making, information management and the application of natural process to environmental remediation. Mr. Rice has acted as Project Director for Chlorinated VOC Historical Case Analysis Initiative, Department of Defense Petroleum Hydrocarbon Cleanup Demonstration Program, and the Revision of California Leaking Underground Fuel Tank Cleanup Process. Mr. Rice is presently the Project Director for evaluating the potential surface and ground water impacts that may result from the use of ethanol as a replacement for MTBE in gasoline. Mr. Rice has authored/coauthored over 50 publications. He is the primary editor and coauthor of the report "Health and Environmental Assessment of the Use of Ethanol as a Fuel Oxygenate" presented to the California Environmental Policy Council in response to executive order D-5-99. He is a member of the World Federation of Scientists Monitoring Panel on Water and Pollution. Mr. Rice has received the Lawrence Livermore National Laboratory Director's Performance Award.

Mr. Rice has a B.A. in Biology and a Life Secondary Education Credential from San Jose State University, and a M.A. in Marine Biology through California State University Moss Landing Marine Science Institute.

Mr. Rice is the Expert Panel Chairman and Primary Point of Contact. His contact information is: (925) 423-5059. His address is 7000 East Ave., L-544, Livermore, CA 945509234. 